NASATM- 84668

NASA Technical Memorandum 84668

NASA-TM-84668 19830017745

\title{
A VISCOUS SHOCK-LAYER FLOWFIELD ANALYSIS BY AN EXPLICIT-IMPLICIT METHOD
}

R.N. Gupta, P.A. Gnoffo,

and R.W. MacCormack

May 1983

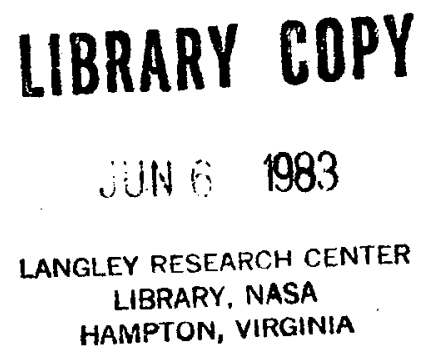

National Aeronautics and
Space Administration

Langley Research Center

Hampton, Virginia 23665 



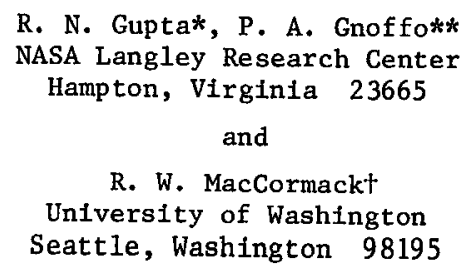

\section{Abstract}

The present work extends the recent1y reported imp1icit analogue of MacCormack's earlier widely used explicit method to external axisymmetric laminar flows with strong entropy gradients. The detalls of the "numerics" of the Implicit part are provided in a body-orlented coordinate system with a moving outer (shock) boundary during the transient part of the solutions. The limiting values of the courant number are obtained when the shock boundary is treated explicitly. The solution algorithm outlined includes the treatment of the source term associated with the equations in weak conservation form. From the results obtained for two sample problems, it becomes clear that accuracy of predictions 1s, indeed, very good at higher values of the courant number. There is a significant saving in overall computing time, depending on the Courant number used and the flow Reynolds number. These properties combined with the simplicity of programing the implicit analog may appeal to researchers for using it in the analysis of 3-D flow problems.

\section{Nomenclature}

$\mathrm{C}_{\mathrm{A}}, \mathrm{C}_{\mathrm{B}}$

constants with values less than or equal to unity

$\mathrm{C}_{\mathrm{f}} \quad$ skin-friction coefficient, $\left(2 \mu_{\mathrm{w}} / \operatorname{Re}\right)(\partial \mathrm{u} / \partial \mathrm{n})_{\mathrm{w}}$

$\mathrm{C}_{\mathrm{H}}$ heat-transfer coefficient, $\left(2 \mu_{W} / \operatorname{Pr} R e\right)(\partial h / \partial n)_{W}$

$\mathrm{CN} \quad$ Courant number

c speed of sound, $\sqrt{\gamma \mathrm{p} / \mathrm{p}}$

$c^{*}$

viscosity, $K$

$\mathrm{H}^{*} / \mathrm{U}_{\infty}^{*} 2$

h nondimensional specific enthalpy, $\mathbf{h}^{*} / \mathrm{U}_{\infty}^{*} 2$

1 finite-difference point in s-direction

$\mathrm{j}$ finite-difference point in n-direction

$k$ count of time steps

$M_{\mathscr{c}} \quad$ freestream Mach number

$\mathrm{M}^{*} \quad$ molecular weight of mixture

$\mathrm{n}$ coordinate direction normal to the body, $\mathrm{n}^{*} / \mathrm{R}_{\mathrm{N}}^{*}$

Pr Prandtl number

p nondimensional pressure, $\mathrm{p}^{*} / \rho_{\infty}^{*} \mathrm{U}_{\infty}^{*}$

*NRC-Senfor Research Associate,

Aerothermodynamics Branch, Space Systems

Division.

**Aero-Space Technologist, Aerothermodynamics

Branch, Space Systems Division.

tProfessor, Dept. of Aeronautics and

Astronautics.

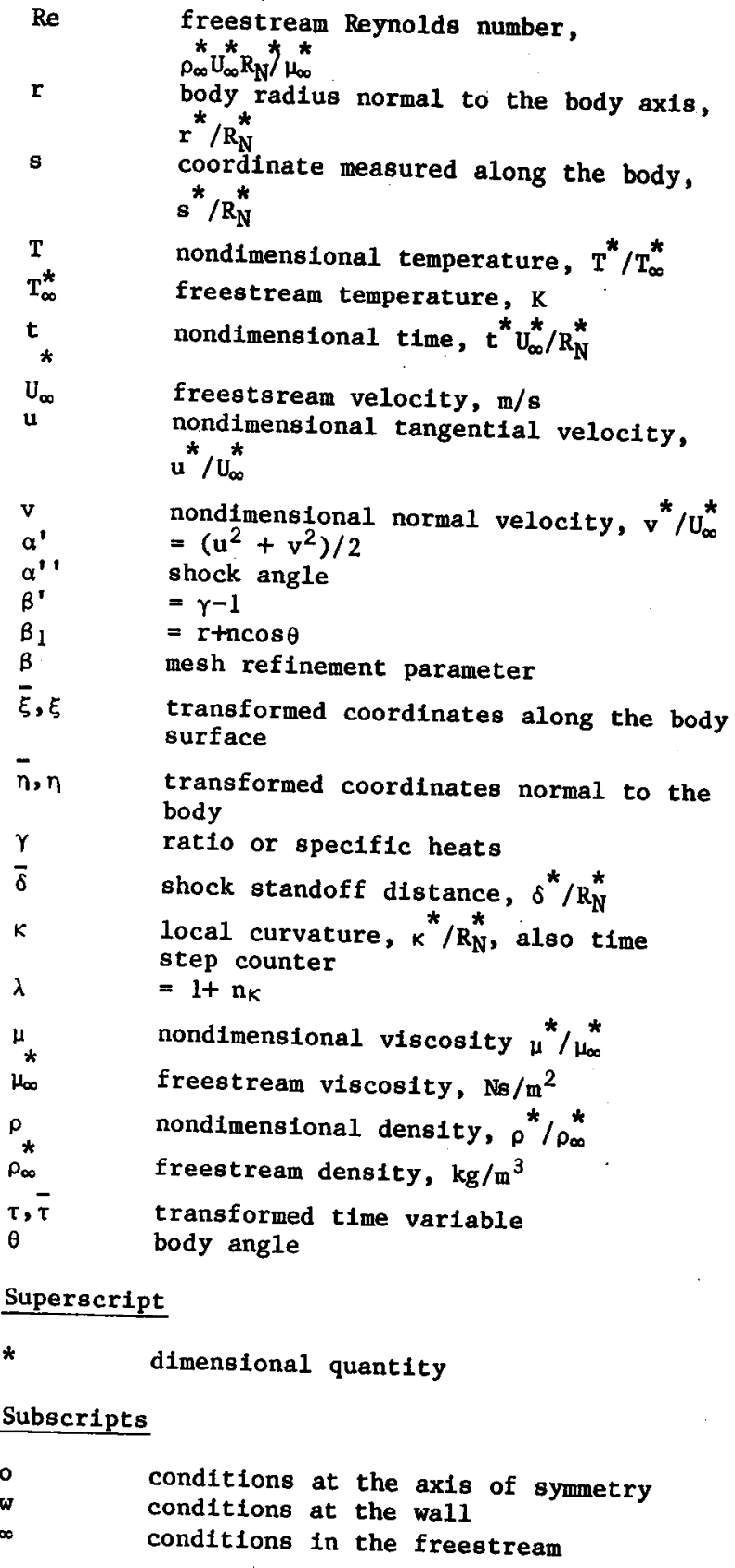

\section{Introduction}

Much progress has been made in the recent past in developing computationally efficient. methods for solving the equations of compressible viscous flow. Foremost among these methods are the implicit time-dependent finite-difference 
techniques ${ }^{1,2,3}$ which are not subject to the conventional stability condition of explicit methods. ${ }^{4}$ However, the application of these techniques is frequently limited by the large computer time per step, their programing complexity, as well as severe accuracy criteria. These Iimitations increase in severity in threedimensional flow analysis. In 1981, MacCormack presented an 1mplicit analog of his earlier widely used explicit method. ${ }^{4}$ One of the basic features of this implicit analog is that it Involves the Inversion of only upper or lower block bidiagonal matrices as opposed to the more costly inversion of block tridiagonal matrices needed in the existing implicit methods.1,2,3 The other major advantage with the method of Ref. 5 is that with more complex problems, on1y the explicit part of the code increases in complexity. The implicit step, which is simply the numerics to obtain enhanced stability with larger values of the Courant numbers, 18 not affected. Since it is easier to program the explicit part even with the increased complexities, the potential for this method is greatly enhanced, espectally for the 3-D flow problems. Moreover, running a program fully explicitly can provide solutions for comparison and check when the implicit analog is used. This is an important feature for the accuracy check not available with the other implicit methods. The Implicit part in the MacCormack's new method is merely an "add-on" to the explicit part. ${ }^{4}$

Recently, Refs. 6, 7, and 8 presented solutions for internal flow problems whereas Ref. 9 provided results for external transonic flows with an integral formulation by using the new Implicit method. References 6 and 8 have basically used the MacCormack's method in Cartesian coordinates as presented in Ref. 5 . The results of Ref. 7 were obtained in more general coordinates $\xi(x, y), n(x, y)$ for a fixed outer boundary and Ref. 9 employed the Cartesian velocity vectors in the solution vector. Except for Ref. 8, the equations solved in these analyses were of strong conservation form. Reference 10 has outlined a procedure for the governing equations which appear in "weak" conservation form. In this form the source terms, which are introduced into the equations by coordinate transformation and/or by turbulence modeling, appear outside the derivatives of the conserved variables.

In the present analysis, MacCormack's implicit analog has been extended to external axisymmetric laminar flows with strong entropy gradients. The matrices involved in the "numerics" of the implicit part have been obtained in a body-oriented coordinate system with a moving outer boundary. The limiting values of the Courant number are provided when the shock boundary is treated explicitly. The method switches automatically from implicit to fully-explicit mode whenever the time step, $\Delta t$, satisfies the explicit stability condition. In general, the method becomes implictt only in regions where the gradients of the flow varlables are large and a refined mesh is needed for higher accuracy.

\section{Analysis}

\section{Flow Governing Equations}

The time-dependent viscous-shock-layer equations employed in the present analysis can be obtained from the unsteady Navier-Stokes equations by keeping terms up to second order in the Inverse square root of the Reynolds number in both the viscous and inviscid regions of the shock layer. These equations, when represented in the body-oriented coordinate system (see Fig. 1) for a perfect gas flow at zero angle of attack, are expressed as ${ }^{11}$

$$
\frac{\partial U}{\partial t}+\frac{\partial M}{\partial s}+\frac{\partial N}{\partial n}+Q=0
$$

where the vectors $U, M, N$, and $Q$ may be obtained by dropping the species-continuity equation and taking the mass fraction of specles and radiative heat flux as zero In Eq. (1) of Ref. 11. These vectors are also given in Ref. 12.

The following limiting form of the governing equations is obtained at the axis of symmetry by differentiating Eq. (1) with respect to 8 and taking a limit as $s+0$ :

$$
\frac{\partial U_{o}}{\partial t}+\frac{\partial M_{o}}{\partial s}+\frac{\partial N_{o}}{\partial n}+Q_{0}=0
$$

where the vectors $U_{0}, M_{0}, N_{0}$, and $Q_{0}$ may once again be obtained from Eq. (2) of Ref. 11 or from Ref. 12 .

The equation of state is given by

$$
\mathrm{p}=\left[\mathrm{R}^{*} \mathrm{~T}^{*} / \mathrm{M}^{*} \mathrm{Ub}_{0}^{*}\right] \rho \mathrm{T}
$$

and the laminar viscosity is obtained from the Sutherland's 1aw

$$
\mu=\left[\left(1+c^{*} / T^{*}\right) /\left(T+c^{*} / T^{*}\right)\right] T^{3 / 2}
$$

\section{Transformation to Computational Plane}

The first of the two independent transformations employed maps the physical domain into a rectangular region in which both the shock and the body are made boundary mesh lines of the computational region. This transformation is

$$
\bar{\tau} \equiv \mathbf{t}, \bar{\xi} \equiv \mathbf{s} \text {, and } \bar{\eta}=\mathbf{1}-\mathbf{n} / \bar{\delta}(\mathrm{s}, \mathrm{t})
$$
are

The transformed forms of Eqs. (1) and (2)

$$
\begin{aligned}
& \frac{\partial \bar{U}}{\partial \bar{\tau}}+\frac{\partial \bar{M}}{\partial \bar{\xi}}+\frac{\partial \bar{N}}{\partial \bar{n}}+\bar{Q}=0 \\
& \partial \bar{U} \\
& \frac{o}{\partial \bar{\tau}}+\frac{\partial \bar{M}}{\partial \bar{\xi}}+\frac{\partial \bar{N}}{\partial \bar{\eta}}+\bar{Q}_{0}=0
\end{aligned}
$$

where

$$
\bar{U}=\mathrm{U} / \mathrm{J}, \overline{\mathrm{M}}=\mathrm{M} \bar{\xi}_{\mathrm{s}} / \mathrm{J}
$$


$\bar{N}=\left(U \bar{n}_{t}+M \bar{n}_{s}+N \bar{n}_{n}\right) / J, \bar{Q}=Q / J$

$\overline{\mathrm{U}}_{\mathrm{O}}=\mathrm{U}_{\mathrm{o}} / \mathrm{J}, \overline{\mathrm{M}}_{\mathrm{O}}=\mathrm{M}_{\mathrm{o}} \bar{\xi}_{\mathrm{s}} / \mathrm{J}$

$\overline{\mathrm{N}}_{0}=\left(\mathrm{U}_{0} \bar{n}_{t}+\mathrm{M}_{\mathrm{o}} \bar{n}_{\mathrm{s}}+\mathrm{N}_{\mathrm{o}} \bar{\eta}_{n}\right) / J, \bar{Q}_{0}=Q_{0} / J$

Here, $J$ is the transformation Jacobian given as

$$
J=\frac{\partial(\bar{\tau}, \bar{\xi}, \bar{\eta})}{\partial(\mathrm{t}, \mathrm{s}, \mathrm{n})}=-\frac{1}{\bar{\delta}}
$$

and $\bar{\xi}_{s}$ denotes $\partial \bar{\xi} / \partial s$, and so forth.

The computation region is next mapped to another plane to allow higher resolution through the viscous layer near the surface. The second transformation 18

$$
\tau \equiv \bar{\tau}, \xi \equiv \bar{\xi} \text {, and } \eta=\frac{\ln \left(\frac{\beta+\bar{\eta}}{\beta-\bar{n}}\right)}{\ln \left(\frac{\beta+1}{\beta-1}\right)}
$$

With this transformation, the final forms of Eqs. (6) and (7) become:

$$
\begin{aligned}
& \frac{\partial \tilde{U}}{\partial \tau}+\frac{\partial \tilde{N}}{\partial \xi}+\frac{\partial \tilde{N}}{\partial \eta}+\mathscr{Q}=0 \\
& \frac{\partial \tilde{U}_{0}}{\partial \tau}+\frac{\partial \tilde{M}_{o}}{\partial \xi}+\frac{\partial \tilde{N}_{o}}{\partial \eta}+\tilde{Q}_{0}=0
\end{aligned}
$$

where

$$
\begin{aligned}
& \tilde{\mathrm{U}}=\overline{\mathrm{U}} / \overline{\mathrm{J}}, \stackrel{\tilde{\mathrm{M}}}{=} \overline{\mathrm{M}} \xi_{\bar{\xi}} / \overline{\mathrm{J}} \\
& \tilde{\mathrm{N}}=\overline{\mathrm{N}} \eta_{\mathrm{n}}-/ \overline{\mathrm{J}}, \tilde{\mathrm{Q}}=\overline{\mathrm{Q}} / \overline{\mathrm{J}}
\end{aligned}
$$

and

$$
\begin{aligned}
& \tilde{\mathrm{U}}_{0}=\overline{\mathrm{U}}_{0} / \overline{\mathrm{J}}, \tilde{\mathrm{M}}_{0}=\overline{\mathrm{M}}_{0} \xi_{\bar{\xi}} / \overline{\mathrm{J}} \\
& \tilde{\mathrm{N}}_{0}=\overrightarrow{\mathrm{N}}_{0} \eta_{\eta} / \overline{\mathrm{J}}, \tilde{Q}_{0}=\overline{\mathrm{Q}}_{0} / \overline{\mathrm{J}}
\end{aligned}
$$

Here, once again, $\bar{J}$ is the transformation Jacobian given as

$$
\bar{J}=\frac{\partial(\tau, \xi, \eta)}{\partial(\bar{\tau}, \bar{\xi}, \bar{\eta})}=\frac{2 \beta}{\ln \left(\frac{\beta+1}{\beta-1}\right)}\left(\frac{1}{\beta^{2}-\bar{\eta}^{2}}\right)
$$

\section{Elements of the Numerical Integration Method}

Since the numerical integration method has been adopted from Ref. 5, the development of the method presented here for a body-fitted coordinate system with the moving outer boundary will not contain the details provided there. The method outlined here will provide details more specific to the problem being analyzed. Equation (10) may be integrated in time by the following implicit predictor-corrector set of finitedifference equations:
For $\mathrm{n}$ odd

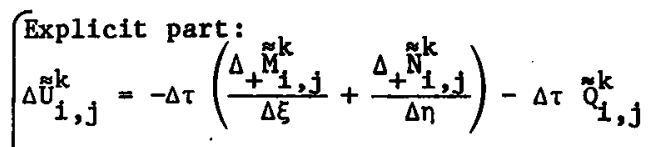

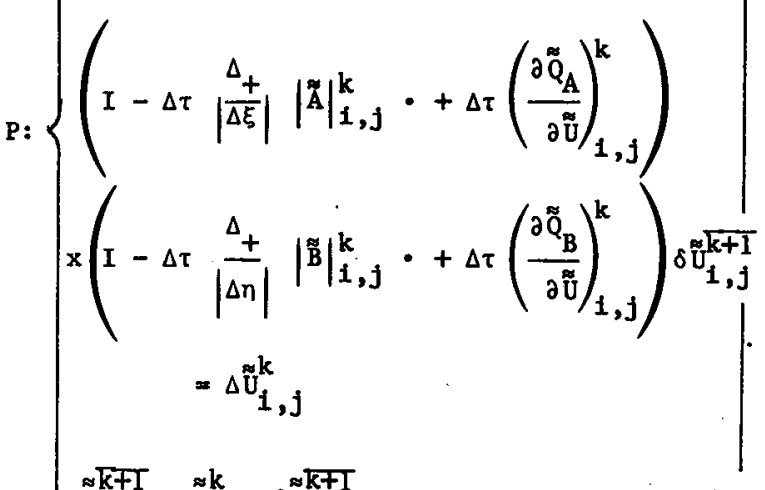

$$
\begin{aligned}
& \left(\tilde{U}_{i, j}^{\bar{K}+1}=\widetilde{U}_{1}^{k}+\delta \tilde{U}_{1, j}^{\bar{k}+1}\right.
\end{aligned}
$$

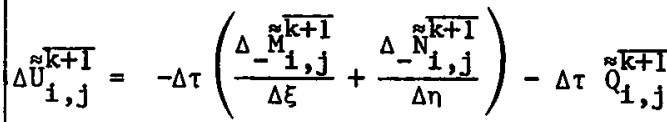

$$
\begin{aligned}
& \text { Implicit part: }
\end{aligned}
$$

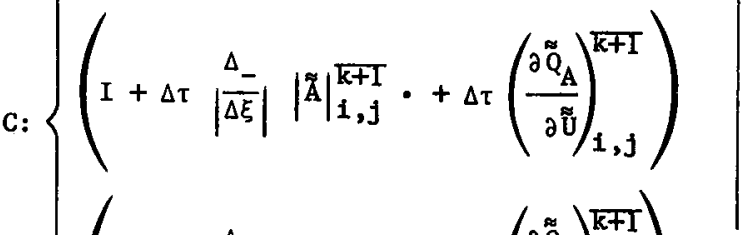

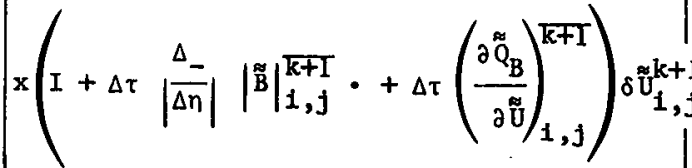

$$
\begin{aligned}
& =\Delta \mathrm{U}_{i, j}^{\mathrm{k}+\mathrm{I}} \\
& \left(\tilde{U}_{1, j}^{k+1}=(1 / 2)\left(\tilde{U}_{1, j}^{k}+\tilde{U}_{i, j}^{k+1}+\delta \tilde{U}_{1, j}^{k+1}\right)\right.
\end{aligned}
$$


And for $n$ even

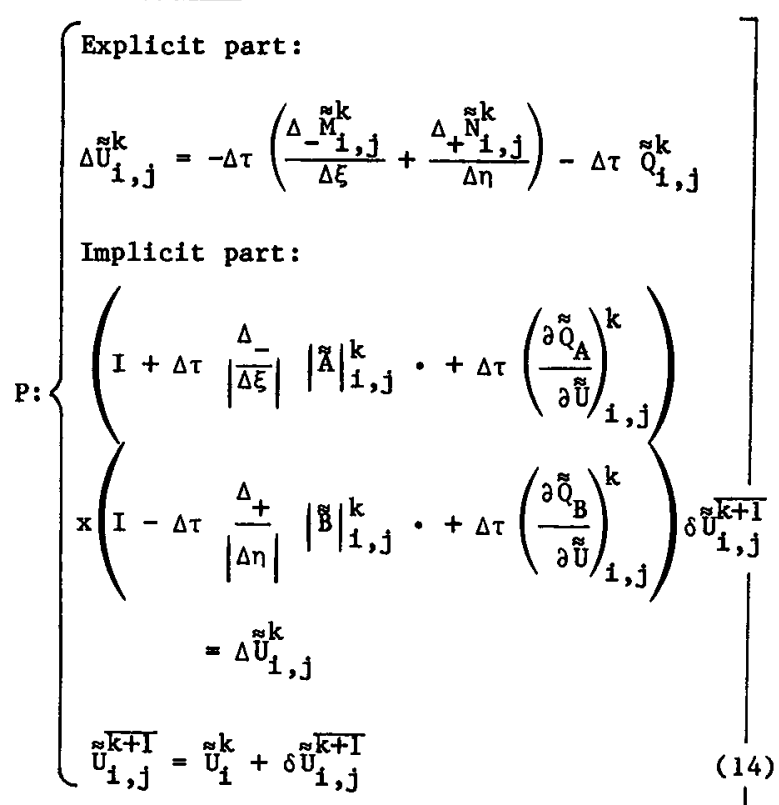

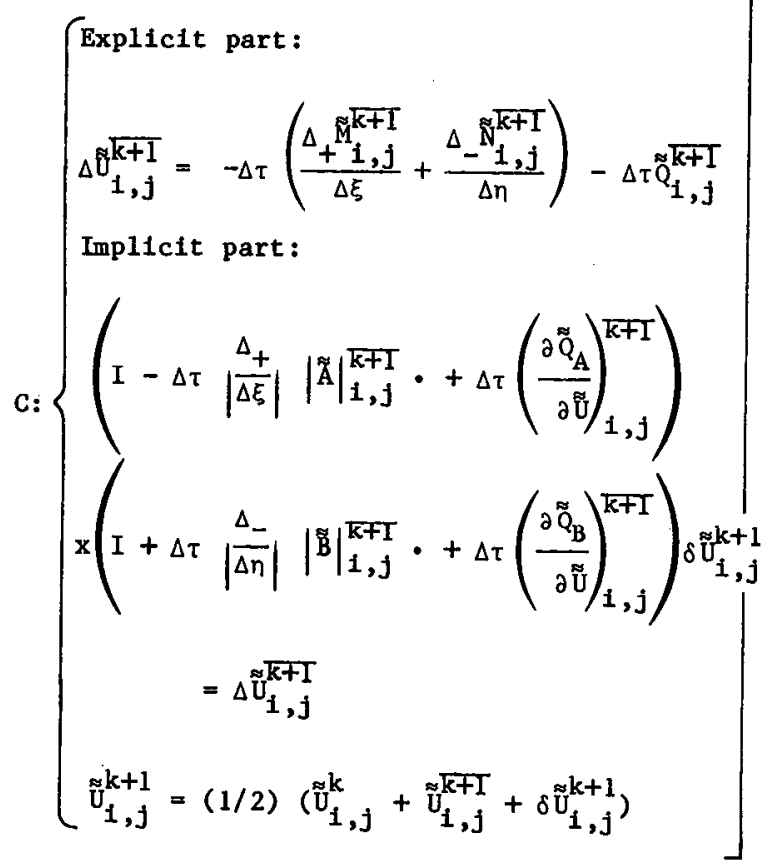

where the source terms $\tilde{Q}_{A}$ and $\tilde{Q}_{B}$ represent parts of the total source identifled with the coordinates $\xi$ and $\eta$, respectively, as discussed in Ref. 10. Based on the definition of $Q$ given in Ref. 12, $\tilde{Q}_{A}$ and $\oint_{B}$ are provided in Appendix A.
It may be noticed from Eqs. (13) and (14) that the order of differencing has been reversed In the streamwise direction (while retaining the same order in the direction normal to the surface) for both the predictor and corrector steps between consecutive time steps. Further, in these equations, $\left(\Delta_{+} / \Delta \xi\right)$ and $\left(\Delta_{+} / \Delta \eta\right)$ are one-sided forward differences and $\left(\Delta_{-} / \Delta \xi\right)$ and $\left(\Delta \_/ \Delta \eta\right)$ are one-sided backward

differences. $|\AA| \widetilde{A} \mid$ and $|\mathbb{B}|$ are matrices with positive eigenvalues and are related to the

Jacobians $\tilde{A}=(\partial \tilde{M} / \partial \tilde{U})$ and $\tilde{B}=(\partial \tilde{N} / \partial \tilde{U})$, respectively, and $I$ is the unit matrix. The dots in the implicit steps indicate that the difference operators $\left(\Delta_{+} /|\Delta \xi|\right),\left(\Delta_{+} /|\Delta \eta|\right)$, etc., apply also to all factors to the right.

Both predictor and corrector consist of two parts: the explicit part solves the governing equations subject to restrictive explicit stability conditions; the implicit part removes these conditions by numerically transforming the equations into an 1mplicit form.

The Jacoblans $\tilde{A}$ and $\mathbb{B}$ are related to the Jacobians $A / \lambda=\partial M / \partial U$ and $B=\partial N / \partial U$ by

$$
\begin{aligned}
& \tilde{A}=I \xi_{t}+\frac{A}{\lambda} \xi_{s}+B \xi_{n} \\
& \tilde{B}=I \eta_{t}+\frac{A}{\lambda} \eta_{s}+B \eta_{n}
\end{aligned}
$$

where $\xi_{t}=\partial \xi / \partial t, \xi_{8}=\partial \xi / \partial s, \xi_{n}=\partial \xi / \partial n$, $n_{t}=\partial n / \partial t, n_{s}=\partial \eta / \partial s$, and $n_{n}=\partial \eta / \partial n$.

The Jacobian matrices $\AA$ and $\tilde{B}$ are provided in Appendix $B$. In the definition of these matrices $\tilde{u}$ and $\tilde{v}$ are the contravarient velocities obtained from

$$
\begin{aligned}
& \tilde{u}=\xi_{t}+\frac{u}{\lambda} \xi_{s}+v \xi_{n} \\
& \tilde{v}=\eta_{t}+\frac{u}{\lambda} n_{s}+v n_{n}
\end{aligned}
$$

Now, the integration method contained in the finite-difference Eqs. (13) and (14) can be

simplified if the matrices $\AA$ and $\bar{B}$ are diagonalized. Knowing the elgenvalue matrices $\Lambda_{A}$ and $\Lambda_{B}$ for $\tilde{A}$ and $\tilde{B}$, respectively, these matrices are diagonalized as:

$$
\begin{aligned}
& \tilde{A}=\tilde{S}_{\xi}^{-1} \Lambda_{A} \tilde{S}_{\xi} \\
& \tilde{B}=\tilde{S}_{\eta}^{-1} \Lambda_{B} \underset{\eta}{\tilde{S}}
\end{aligned}
$$

where $\tilde{S}_{\xi}$ and $\tilde{S}_{\eta}$ are the efgenvector matrices of $\tilde{A}$ and $\tilde{B}$ and $\tilde{S}_{\xi}^{-1}$ and $\tilde{S}_{\eta}^{-1}$ are the inverses of 
$\xi_{\xi}$ and $\S_{\eta}$, respectively. The complete definition of these matrices is given in Appendix $C$.

The diagonal matrices $\Lambda_{A}$ and $\Lambda_{B}$ in Eq. (16), formed from the eigenvalues of $\AA$ and $\tilde{B}$, respectively, may be written down as:

$$
\begin{aligned}
& \Lambda_{\mathrm{A}}=\left[\begin{array}{llll}
\sigma_{\mathrm{A} 1} & 0 & 0 & 0 \\
0 & \sigma_{\mathrm{A} 2} & 0 & 0 \\
0 & 0 & \sigma_{\mathrm{A} 3} & 0 \\
0 & 0 & 0 & \sigma_{\mathrm{A} 4}
\end{array}\right] \\
& \Lambda_{\mathrm{B}}=\left[\begin{array}{llll}
\sigma_{\mathrm{B} 1} & 0 & 0 & 0 \\
0 & \sigma_{\mathrm{B} 2} & 0 & 0 \\
0 & 0 & \sigma_{\mathrm{B} 3} & 0 \\
0 & 0 & 0 & \sigma_{\mathrm{B} 4}
\end{array}\right]
\end{aligned}
$$

where

$$
\begin{aligned}
& \sigma_{\mathrm{A} 1}=\stackrel{\approx}{\mathrm{u}}, \sigma_{\mathrm{A} 2}=\stackrel{\approx}{\mathrm{u}}+\mathrm{d}, \sigma_{\mathrm{A} 3}=\tilde{\mathrm{u}}, \sigma_{\mathrm{A} 4}=\stackrel{\approx}{\mathrm{u}}-\mathrm{d} \\
& \sigma_{\mathrm{B} 1}=\stackrel{\approx}{\mathrm{v}} ; \sigma_{\mathrm{B} 2}=\stackrel{\approx}{\mathrm{v}}, \sigma_{\mathrm{B} 3}=\stackrel{\approx}{\mathrm{v}}+\mathrm{g}, \sigma_{\mathrm{B} 4}=\stackrel{\approx}{\mathrm{v}}-\mathrm{g}
\end{aligned}
$$

with

$$
\begin{aligned}
& d=c \sqrt{\left(\xi_{s} / \lambda\right)^{2}+\left(\xi_{n}\right)^{2}} \\
& g=c \sqrt{\left(n_{s} / \lambda\right)^{2}+\left(n_{n}\right)^{2}}
\end{aligned}
$$

The matrices $|\tilde{A}|$ and $|\widetilde{B}|$ appearing in Eqs. (13) and (14) may now be formed by replacing the matrices $\Lambda_{A}$ and $\Lambda_{B}$ by positively-valued diagonal matrices $\mathrm{D}_{\mathrm{A}}$ and $\mathrm{D}_{\mathrm{B}}$. The matrices $|\tilde{A}|$ and $|\tilde{B}|$ are thus defined as

$$
\begin{aligned}
& |\tilde{A}|=\tilde{S}_{\xi}^{-1} D_{A} \tilde{S}_{\xi} \\
& |\tilde{B}|=\tilde{S}_{\eta}^{-1} D_{B} \frac{\tilde{S}}{\eta}
\end{aligned}
$$

where

$$
\begin{aligned}
& \mathrm{D}_{\mathrm{A}}=\max \left(\left|\Lambda_{\mathrm{A}}\right|+\tilde{\lambda}_{\mathrm{A}} \mathrm{I}, 0.0\right) \\
& \mathrm{D}_{\mathrm{B}}=\max \left(\left|\Lambda_{\mathrm{B}}\right|+\tilde{\lambda}_{\mathrm{B}} \mathrm{I}, 0.0\right)
\end{aligned}
$$

and

$$
\begin{aligned}
& \tilde{\lambda}_{A}=\frac{2 \nu}{\rho \Delta \xi}\left[\left(\frac{\xi_{s}}{\lambda}\right)^{2}+\xi_{n}^{2}\right]-c_{A} \frac{\Delta \xi}{\Delta \tau} \\
& \tilde{\lambda}_{B}=\frac{2 \nu}{\rho \Delta n}\left[\left(\frac{n}{s}\right)^{2}+\eta_{n}^{2}\right]-C_{B} \frac{\Delta n}{\Delta \tau}
\end{aligned}
$$

with $\tilde{v}=\max \left\{\frac{4}{3} \frac{\mu}{\operatorname{Re}}, \frac{\mu}{\operatorname{Re}} \frac{\gamma}{\operatorname{Pr}}\right\}$

The constants $C_{A}$ and $C_{B}$ are related to the Courant number used for explictt stability. The Courant number, $\mathrm{CN}$, is related to the maximum time step $\Delta \tau$ permitted in the $n$-direction, for example, through the Courant-Friedrichs-Lewy (CFL) criterion

$$
\Delta \tau \leq \mathrm{CN} \frac{\Delta n}{|\vec{v}|+g+\frac{2 \gamma \mu}{\rho \operatorname{RePr} \Delta \eta}\left\{\left(\frac{n_{s}}{\lambda}\right)^{2}+n_{n}^{2}\right\}}
$$

Here $C_{N}$ is less than or equal to unfty for an explicit stable solution.

Following Ref. 10, a procedure can also be outlined for obtaining the Jacoblans ( $\left.\partial \tilde{Q}_{A} / \partial \partial^{\circ} U\right)$ and $\left(\partial \tilde{Q}_{B} / \partial \tilde{U}\right)$ of the source term $\tilde{Q}\left(=\tilde{Q}_{A}+\tilde{Q}_{B}\right)$ with respect to U. Justification of this procedure is provided in Ref. 10.

$$
\text { Let } \Phi_{A}=\Delta \tau \quad \frac{\partial \tilde{Q}_{A}}{\partial \tilde{U}}
$$

$$
\text { and } \Phi_{B}=\Delta \tau \frac{\partial \tilde{Q}_{B}}{\partial \tilde{U}}
$$

We now define the scalar matrices $\left|\Phi_{A}\right|$ and $\left|\Phi_{B}\right|$ as follows

$$
\begin{aligned}
& \left|\Phi_{A}\right|=\phi_{A} I \\
& \left|\Phi_{B}\right|=\phi_{B} I
\end{aligned}
$$

Here $\phi_{A}$ and $\phi_{B}$ are obtained from ${ }^{9}$

$$
\begin{gathered}
\phi_{A} \geq \max \left(\stackrel{\approx}{q}_{A_{0}}-\Delta \tau \tilde{\rho}_{A^{\prime}}, 0.0\right) \\
\phi_{B} \geq \max \left(\tilde{q}_{B_{O}}-\Delta \tau \tilde{\rho}_{B}, 0.0\right)
\end{gathered}
$$

with

$$
\begin{aligned}
& \tilde{\mathrm{q}}_{\mathrm{A}_{0}}=\left\{\max \frac{\Delta \tau}{\Delta \xi}\left(\sigma_{A_{\max }}+\tilde{\lambda}_{A}\right), 0.0\right\} \\
& \tilde{\mathrm{q}}_{\mathrm{B}_{\mathrm{O}}}=\left\{\max \frac{\Delta \tau}{\Delta \eta}\left(\sigma_{B_{\max }}+\tilde{\lambda}_{B}\right), 0.0\right\}
\end{aligned}
$$

and

$$
\left.\begin{array}{l}
\tilde{\rho}_{A}=\max _{j}\left|\hat{\lambda}_{A j}\right| \\
\tilde{\rho}_{B}=\max _{j}\left|\hat{\lambda}_{B j}\right|
\end{array}\right\} \quad j=1,2,3,4
$$

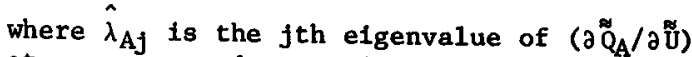

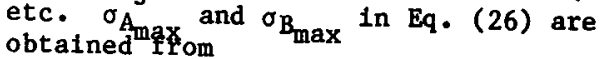

$$
\left.\begin{array}{l}
\sigma_{A_{\max }}=\max _{j}\left|\sigma_{A j}\right| \\
\sigma_{B_{\max }}=\max _{j}\left|\sigma_{B j}\right|
\end{array}\right\} j=1,2,3,4 .
$$


and $\sigma_{A j}$ and $\sigma_{B j}$ are defined following

Eq. (18), whereas $\tilde{\lambda}_{A}$ and $\tilde{\lambda}_{B}$ are given by

Eq. (21).

The Constants $C_{A}$ and $C_{B}$ appearing in Eq. (21) are assigned a value of $1 / 2$ in Ref. 5 . However, if the time step $\Delta \tau$ in the expressions for $\tilde{\lambda}_{A}$ and $\tilde{\lambda}_{B}$ is a local minimum, a value of up to unity may be used for $C_{A}$ and $C_{B}$ to speed up the calculations.

\section{Solution Algorithm and Boundary Conditions}

As shown in expression (20) for the diagonal matrices $D_{A}$ and $D_{B}$, their elements are non-negative. Whenever the elements become negative, they are replaced by zero. This implies that the CFL condition, Eq. (22), is

satisfied and matrices $|\tilde{A}|$ and $|\tilde{B}|$ become null matrices. For this case the impilcit portion of the scheme contained in Eqs. (13) and (14) w11l be bypassed. For the flow regions where the CFL condition is not satisfied, the implicit parts in Eqs. (13) require the solution of upper-block bidiagonal system of equations for the predictor step and the solution of lower-block bidlagonal system of equations for the corrector step, etc. The integration scheme, for the case where the CFL criterion is not satisfied, can be illustrated by solving the predictor part of Eq. (13) as detailed in the subsequent paragraphs.

In this algorithm we replace the matrices $\Phi_{\mathrm{A}}$ and $\Phi_{\mathrm{B}}$ (defined in $\mathrm{Eq} \cdot(23)$ ) by the scalar matrices $\left|\Phi_{\mathrm{A}}\right|$ and $\left|\Phi_{\mathrm{B}}\right|$ provided by Eqs. (24) and $(25)$

$$
\begin{aligned}
\text { Let } \delta \mathrm{U}_{i, j}^{*}= & \left(I-\Delta \tau\left|\frac{\Delta_{+}}{\Delta \eta}\right||\mathrm{B}|_{i, j}^{k} \cdot+\left|\Phi_{B_{i, j}}^{k}\right|\right) \\
& x \delta \tilde{U}_{i, j}^{\overline{k+1}}
\end{aligned}
$$

then, the predictor step becomes

$$
\begin{aligned}
& \left(I+\left|\frac{\Delta \tau}{\Delta \xi}\right||\tilde{A}|_{1, j}^{k}+\left|\Phi_{A_{i, j}}^{k}\right|\right) \delta \tilde{U}_{1, j}^{*}=\Delta \tilde{U}_{i, j}^{k} \\
& +\left|\frac{\Delta \tau}{\Delta \xi}\right||\AA|_{1+1, j}^{k} \delta \tilde{U}_{i+1, j}^{*}
\end{aligned}
$$

an upper-bidiagonal equation and the solution can be obtained for each $j$ by sweeping in the decreasing $i$ direction.

After obtaining $\delta \mathrm{U}_{1, j}^{*}$ for all $1, j$, then

$$
\begin{aligned}
& \left(I+\left.\left.\left|\frac{\Delta \tau}{\Delta \eta}\right|\right|^{\tilde{B}}\right|_{i, j} ^{k}+\left|\Phi_{B_{i, j}}^{k}\right|\right) \delta U_{1, j}^{\delta k+I}=\delta \tilde{U}_{i, j}^{*} \\
& +\left.\left.\left|\frac{\Delta \tau}{\Delta \eta}\right|\right|^{\mathbb{B}}\right|_{i, j+1} ^{k} \delta \tilde{U}_{1, j+1}^{\overline{k+1}}
\end{aligned}
$$

This equation is also upper-bidiagonal and is solved for each $i$ by sweeping in the decreasing $j$ direction. This gives $\delta U_{i, j}^{k+1}$ for all $1, j$. Then

$$
\tilde{U}_{i, j}^{k+1}=\tilde{U}_{i, j}^{k}+\delta \tilde{U}_{1, j}^{k+I} \text { etc. }
$$

For understanding the present method, let us examine the procedure for solving the blockbidlagonal Eq. (30) for the $n$-coordinate or $j$-direction. If we define

$$
w_{j}=\delta \tilde{U}_{i, j}^{*}+\left.\left.\left|\frac{\Delta \tau}{\Delta \eta}\right|\right|^{\tilde{B}}\right|_{i, j+1} ^{k} \delta \tilde{U}_{i, j+1}^{k+T}
$$

the Eq. (30), after some matrix multiplication, may be written as

$$
\begin{gathered}
\delta \tilde{U}_{i, j}^{k+1}=\left(\tilde{s}_{n}^{-1}\right)_{1, j}^{k}\left\{\left(1+\phi_{B_{i, j}^{k}}^{k}\right) I+\left|\frac{\Delta \tau}{\Delta \eta}\right|_{B_{i, j}^{k}}^{k}\right\}^{-1} \\
x\left(s_{n}\right)_{i, j}^{k} w_{j}
\end{gathered}
$$

where we have substituted for $|\tilde{B}|$ from Eq. (19) and employed the relation $\left|\Phi_{B}\right|=\phi_{B} I$ for the scalar matrix $\left|\Phi_{B}\right|$.

The integration procedure in the Implicit part begins with the vectors $\mathrm{U}_{1, j}^{k}$, given for al1 $1=1,2, \ldots, I$ and $j=1,2, \ldots, J$; $\delta \tilde{U}_{1, j}^{*}$ given for all $1=2,3, \ldots, I-1$ and $j=$ $2,3, \ldots, \mathrm{J}-1$ and $|\mathbb{B}|_{1, J} \delta U_{i, J}^{K+T}$ given for all $1=2,3, \ldots, I-1$. The quantity $\left.\left.\right|_{B} ^{\infty}\right|_{1, j} ^{k} \delta U_{1, J}^{k+I}$ represents the flux of change that crosses the top mesh boundary. If this boundary is located in the far flowfield or if the mesh is stretched so that $\Delta \tau$ satisfies the local explicit stability condition (22) at the mesh points near the boundary, as in the case of the test problems to be discussed later, this flux is set equal to zero. Otherwise, it should be suitably specified from the boundary conditions.

Following Ref. 5, the solution algorithm may now be summarized for each $i$ and for $\mathrm{J}=\mathrm{J}-1$, $\mathrm{J}-2, \ldots, 3,2$ in the following seven steps:

1) $w_{j}=\delta U_{1, j}^{*}+\left\{\frac{(|\Delta \eta| / \Delta \tau)_{1, j+1}}{(|\Delta \eta| / \Delta \tau)_{1, j}}\right\}$

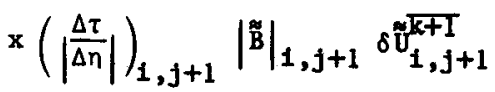

2) $x_{j}=\left(\tilde{s}_{\eta}\right)_{i, j}^{k} w_{j}$

3) $\mathrm{D}_{\mathrm{B}_{1, j}}^{\mathrm{k}}=\max \left\{\left|\Lambda_{\mathrm{B}_{1, j}}^{\mathrm{k}}\right|+\lambda_{\mathrm{B}_{1, j}}^{\mathrm{k}} I, 0.0\right\}$ 
4) $Y_{j}=\left.\left.\left|\left(1+\phi_{B_{i, j}^{k}}^{k}\right) I+\right| \frac{\Delta \tau}{\Delta n}\right|_{i, j} D_{B_{i, j}}^{k}\right|^{-1} x_{j}$

5) $\delta \mathrm{U}_{1, j}^{\mathrm{k}+\mathrm{I}}=\left(\tilde{s}_{n}^{-1}\right)_{i, j}^{k} \mathrm{Y}_{j}$

6) $z_{j}=D_{B_{i, j}}^{k} Y_{j}$

7) $\left(\left|\frac{\Delta \tau}{\Delta \eta}\right|\right)_{i, j}|\tilde{B}|_{i, j}^{k} \delta \tilde{U}_{i, j}^{k+1}=\left(\left|\frac{\Delta \tau}{\Delta \eta}\right|\right)_{i, j}\left(\tilde{s}_{n}^{-1}\right)_{i, j}^{k} z_{j}$

The matrix inversion of step 4 is trivial because the matrix $D_{B}$ is diagonal. The solution at grid point $(i, j)$ is obtained at step 5 , and the flux to be used at grid point $(1, j-1)$ is obtained at step 7. In computational plane $(|\Delta n|)_{i, j+1}=(|\Delta n|)_{i, j}=|\Delta n|$ and, if global minimum time step is used, $(\Delta \tau)_{1, j+1}=$ $(\Delta \tau)_{i, j}=\Delta \tau$. For this case $W_{1, j}$ in step 1 is obtained from

$$
W_{j}=\delta \tilde{U}_{1, j}^{*}+\left|\frac{\Delta \tau}{\Delta \eta}\right||\tilde{B}|_{i, j+1}^{k} \delta \tilde{U}_{i, j+1}^{\bar{k}+1}
$$

However, if a local minimum time step is employed in the computational plane (with $(|\Delta \eta|)_{i, j+1}=$ $(|\Delta n|), w_{j}$ may be obtained from

$$
\begin{aligned}
W_{j} & =\delta \tilde{U}_{1, j}^{*}+\frac{(\Delta \tau)_{1, j}}{(\Delta \tau)_{1, j+1}} \\
& x\left(\left|\frac{\Delta \tau}{\Delta n}\right|\right)_{1, j+1}||_{i, j+1}^{\tilde{B}} \delta U_{1, j+1}^{k} \overline{k+1}
\end{aligned}
$$

For the boundary condition required at the solid wall boundary, the computed end flux terms $|\tilde{B}| \delta \tilde{U}_{i}^{k+1}$, are saved for use as boundary condition for the corrector step that sweeps away from this boundary in the increasing $j$-direction. Using the reflection principle for the wall placed between the first and second grid point, the starting flux for the corrector step is obtained from

$$
|\tilde{B}|_{i, 1}^{\bar{k}+1} \delta \tilde{U}_{1,1}^{k+1}=\left.\left.E\right|_{B} ^{\tilde{B}}\right|_{i, 2} ^{k} \delta \tilde{U}_{i, 2}^{k+1}
$$

where

$$
\mathbf{E}=\left[\begin{array}{llrl}
1 & 0 & 0 & 0 \\
0 & 1 & 0 & 0 \\
0 & 0 & -1 & 0 \\
0 & 0 & 0 & 1
\end{array}\right]
$$

This condition ensures that the net mass, tangential momentum, and energy fluxes transmitted across the wall vanish and that the net transverse momentum at the solid wall remains

zero between $\overline{k+1}$ and $k+1$ time steps.

In the present work the Courant number employed in the $\xi$-direction was always less than unity due to the large mesh size employed in that direction. Accordingly, purely-explicit boundary conditions are used in the $\xi$-direction, implying

the end flux terms $|\bar{A}|_{1, j}^{k} \delta \tilde{U}_{1}^{k+1},|\tilde{A}|_{I, j}^{k} \cdots \overline{k+1}$ $\delta \mathrm{U}_{\mathrm{I}, \mathrm{j}}$ etc. to be zeto. 'In fact, In this case the Implicit part in the $\xi$-direction 18 bypassed.

The explicit boundary conditions employed are no slip at surface, no surface mass transfer, a specified wall temperature, and pressure at the wall is assumed to be equal to the pressure at the adjacent grid point in the normal direction.

The Rankine-Hugonlot relations are employed for the explicit outer-boundary conditions to obtain flow properties Immediately behind the shock. These relations in the body-oriented coordinate system are provided in Ref. 13. The flow conditions along the supersonic downstream boundary are obtained by extrapolation from the upstream grid points.

\section{Artificial Damping}

A fourth-order damping ${ }^{14}$ is used in the explicit part of the corrector step in Eqs. (13) and (14) for obtaining stable solutions over a large number of time steps. The following damping term is used in both the predictor and corrector steps with the impliclt parts:

$$
T_{i, j}^{k}=\frac{\left\{\left|x_{1}\right|\right\}^{k}}{\left(\left|\frac{\Delta \tau}{\Delta \eta}\right|\right)_{, i, j}\left(\frac{\gamma-1}{\gamma}\right)_{\rho}}
$$

This term is evaluated during step 3 , of the solution algorithm given in the previous section, using the first element of the vector $x$. Accordingly, $D_{B}$ in step 3 is obtalned from

$$
D_{B_{i, j}}^{k}=\max \left\{\left|\Lambda_{B_{1, j}}^{k}\right|+\lambda_{B_{i, j}}^{k} I+T_{i, j}^{k} I, 0.0\right\}
$$

As steady state is approached $\left|x_{1}\right|$ approaches zero and the added term vanishes.

\section{Discussion of Results}

The numerical method presented here has been applied to two test problems, both involving the analysis of viscous-shock-layer equations in the body-oriented coordinate system. These two examples are taken from Refs, 12 and 15 and provide fairly severe viscous-shock-layer flow fields for testing the present method. The main difference between the two test problems is that the first one (taken from Ref. 12) is characterized by a reference Reynolds number (based on nose radius and freestream conditions) with a value of about $1.57 \times 10^{5}$, whereas the second problem 15 has a reference Reynolds number around $1.23 \times 10^{6}$. The flow conditions of Ref. 15 are considered typical of the Jovian entry conditions. The reference Reynolds number mentioned here is related 16 to the mesh Reynolds number and provides a criterion by which the mesh near solid-surface boundaries may be refined.

Problem I - (Ref. 12)

Probe geometry: $45^{\circ}$ half-angle spherically-blunted cone with a nose radius $\left(\mathrm{R}_{\mathrm{N}}{ }^{*}\right)$ of $0.222 \mathrm{~m}$. 
Jovian atmosphere: Hydrogen-hellum mixture $\left(0.90 \mathrm{H}_{2}+0.10 \mathrm{He}\right)$ under perfect-gas conditions.

Other flow field parameters: $M_{\infty}=43.84$, $\mathrm{T}_{\infty}{ }^{*}=145 \mathrm{~K}, \rho_{\infty}{ }^{*}=1.27 \times 10^{-4} \mathrm{~kg} / \mathrm{m}^{3}$, $\mathrm{T}_{\mathrm{w}}{ }^{*}=4000 \mathrm{~K}, \gamma=1.224, \mathrm{R}_{\mathrm{g}}{ }^{*}=3593.6$ $\mathrm{J} \mathrm{kg}^{-1} \mathrm{~K}^{-1}$, Re $=1.567 \times 10^{5}, \mathrm{Pr}=0.72$.

Problem II - (Ref. 15)

Probe geometry: $44.25^{\circ}$ half-angle spherecone with a nose radius $\left(\mathrm{R}_{\mathrm{N}}{ }^{*}\right)$ of $0.352 \mathrm{~m}$.

Jovian atmosphere: Orton nominal atmosphere of hydrogen-helium mixture $\left(0.895 \mathrm{H}_{2}+0.105\right.$ He) under perfect-gas assumption.

Other flowfield parameters: $M_{\infty}=43.76$, $T_{\infty}^{*}=151.2 \mathrm{~K}, \rho_{\infty}^{*}=4.966 \times 10^{-4} \mathrm{~kg} / \mathrm{m}^{3}$, $\mathrm{T}_{\mathrm{w}}{ }^{*}=4022,80 \mathrm{~K}, \mathrm{\gamma}=1.217, \mathrm{R}_{\mathrm{g}}{ }^{*}=3737.45$ $\mathrm{J} \mathrm{kg}^{-1} \mathrm{~K}^{-1}, \operatorname{Re}=1.227 \times 10^{6}, \operatorname{Pr}=0.72$.

Through the transformations of Eqs. (5) and (9), the physical domain, shown in Fig. 1, is transformed into a computational domain with equally spaced grids in both the directions, along and normal to the body surface. Parameter $B$ In Eq. (9) controls the amount of grid refinement desired near the wall in the physical domain, with values near 1 giving the largest amount of refinement. However, the mesh refinement 18 done only to the point at which the mesh Reynolds number reaches the order of unity 10,16 . At this point, diffusion and convection processes are equally resolved. To go beyond this point to smaller inesh sizes and hence to lower inesh Reynolds numbers $\dagger$, one would arrive at a mesh scale at which diffusion dominates. For such problems, more complex and timeconsuming methods $1,2,3$ involving tridlagonalinversion procedures should be used. In the present calculations, the value of $\beta$ was chosen to obtain the mesh keynolds number ${ }^{16}$ of order unity and $\mu \Delta \tau / \rho(\Delta \eta)^{2}$ was kept less than $1 / 2$ to avoldany possible steady-state solution dependence on $\Delta \tau$. This was done by reducing the time step near the end of the calculation. The damping coefficient ${ }^{14} \varepsilon$ used with the explicit part was also reduced with the reduction in $\Delta \tau$ so as to permit comparisons between the solutions having simflar amounts of artifictal damping. The damping term assoclated with the $1 \mathrm{mplicit}$ part goes to zero as steady state is approached.

The varlous results presented here have been obtained for the values of the Courant number (CN) ranging from 1 to 15 . The maximum value of the courant number, which may be used without the

FAs pointed out in Ref. 10 also, there is a restriction in the present method on the manner in which, for example, $\Delta \tau$ and $\Delta \eta$ go to zero in a mesh-refinement procedure. The restriction requires that $\mu \Delta \tau / \rho(\Delta \eta)^{2}$ remain bounded as $\Delta \tau$ and $\Delta \eta+0$. With this restriction, $\Delta \tau-\rho(\Delta n)^{2} / \mu-$ $O(\Delta \eta)^{2}$. This limitation on $\Delta \tau$ is a nuisance which one would like to avold. However, this is the price pald for using a simple bidiagonal Inversion in place of the more complex tridiagonal-inversion procedures for the viscous terms. specification of boundary values for the Implicit part near the shock, is limited by the relation

$$
\mathrm{CN} \leq 0.75 \frac{\left[\mathrm{c}_{B}\left(\Delta \mathrm{t}_{\ell}\right)_{n=n_{B}}\right.}{\left(\Delta \mathrm{t}_{\ell}\right)_{n=0}}
$$

where $C_{B}$ has also been used in Eq. (21) and can have a value of unity or less. The local minimum time step near the surface $\left(\Delta t_{\ell}\right)_{n=0}$, used in the above relation, depends on the mesh size employed there. Therefore, in this case where the shock is treated explicitly and the mesh size near the surface 18 established by requiring that the mesh Reynolds number be unity, there would be an upper 1 Imit on the value of $\mathrm{CN}$ which may be used with the present method. However, this is not a limitation of the method. If the shock boundary can be treated implicitly, values larger than the limting value of the Courant number Indicated here may be used. In fact, the present method is unconditionally stable if the flux boundary condition $|\tilde{b}| \delta \tilde{U}$ (see paragraph following Eq. (32)) can be evaluated implicitiy. It represents the implicit part of the boundary conditions. Its evaluation by such means as lagging in time, etc. will limt the stablitity of the present method to smaller Courant numbers as experienced in Refs. 6 and 7.

Figure 2 gives varlous time steps which may be employed at a given body station. The time step shown by curve 2 has been used for CN < 1 (employing the explicit method ${ }^{4}$ ), whereas curve 4 has been employed with the implicit analog ${ }^{5}$ for $C N>1$. T1me step $\Delta t_{1}$ shown by curve 2 is defined as

$$
\Delta t_{1}=(1-0.0025 \mathrm{j}) \Delta \mathrm{t}_{\ell} ; \mathrm{j}=1,2, \ldots, 100
$$

where $\Delta t_{\ell}$ is the local minimum time step shown by curve 1 and $j$ is the mesh point counter with a value of 1 at the wall $(n=0)$ and a value of 100 just behind the shock. If a very large value of the Courant number 18 used with the implicit analogue, a time step shown by curve 5 would result and cause the calculations to go implictt from the wall to the shock. The global minimum curve 3 and the fully-implicit curve 5 have not been used in the present work and are included for illustration only. It becomes clear from this figure that, for finer mesh resolution near the surface, larger values of the Courant number can be used without needing to specify the shock-boundary condition implicitly.

A $101 \times 15$ mesh size has been used in the present computations with 101 mesh points in the direction normal to the surface. The mesh points along the body were evenly spaced at $\Delta F_{2}(\equiv \Delta s)$ values of 0.1963 for problem $I$ and at $\Delta \xi$ values of 0.1597 for problem II. The solution is considered as converged to the steady-state value when the following criterion lo satistied:

$$
\underset{1}{\operatorname{Maximum}}||\left(C_{H}\right)_{i}^{K}-\left(C_{H}\right)_{1}^{K-50} J /\left(C_{H}\right)_{I}^{K} \mid<\varepsilon^{*} ;
$$

where $\epsilon^{*}=0\left(10^{-2}\right)$ and $C_{H 1} 18$ the nondimensional heat-transfer coeffictent defined as 


\section{$C_{H}=\frac{2 \mu w}{\operatorname{Pr} \operatorname{Re}}\left(\frac{\partial h}{\partial n}\right)$}

The convergence test given here is for $\mathrm{CN}=10$ and is made every 50 time steps; for $\mathrm{CN}=1$ it is made every 500 time steps, etc.

During the analysis it was found that the quality and stability of the solutions improved if the order of differencing along the streamwise direction is reversed from one time step to the next time step in both predictor and corrector steps as given in Eqs. (13) and (14).

The solution algorithm outlined in this work includes the treatment of the source term in the implicit parts of the method. However, in the results obtained here, the source term in implicit parts of Eqs. (13) and (14) was neglected, 1mplying that $\Delta \tau\left(\partial \tilde{Q}_{A} / \partial \tilde{U}\right)$ and $\Delta \tau\left(\partial \tilde{Q}_{B} / \partial \tilde{U}\right)$, etc. were small enough. The observation of Ref. 8 in this regard appears to be true for the two problems treated here. The physics of the problem is contained in the explicit parts of Eqs. (13) and (14) and the source term is retained there. Dropping the source term from the numerics contained in the implicit parts of these equations does not seem to affect the results appreciably. The inclusion of the source term in the 1mplicit part as outlined here is likely to increase the computational time per time step by an estimated 5 to 10 percent.

The computed results for problem I are given in Figs. 3 to 5 . These figures contain results for Courant number (CN) of 1 and 5 with the mesh refinement parameter $\beta$ having a value of 1.1 . The $C N=1$ results are those of Ref, 12 and have been recomputed here. The two factors outlined earlier prevented obtaining results for higher values of $\mathrm{CN}$. The requirement of keeping the mesh Reynolds number around unity did not allow further mesh refinement, whereas the specification of the shock boundary condition explicitiy with the given amount of mesh refinement did not permit the use of higher values of $\mathrm{CN}$ for this problem.

Figure 3 shows the shock stand-off distance along the body surface. There is a very good agreement between the two values predicted by using $\mathrm{CN}=1$ and 5 over the entire body surface. The velocity and temperature profiles of Fig. 4 show a similar agreement, the comparison being somewhat superior over the spherical portion (Fig. 4(a)). The comparison between the predicted values of skin-friction and heattransfer coefficients and surface pressure for $\mathrm{CN}=1$ and 5 is given in Fig. 5. Once again, the various distributions compare very well at the two $\mathrm{CN}$ values.

Figures 6 through 8 contain results for sample problem II. As pointed out earlier, the reference Reynolds number in this case is almost an order of magnitude larger than the one for problem I. Accordingly, a finer mesh $(\beta=1.02)$ can be employed here, still keeping the mesh Reynolds number of order unity. This allows the use of a higher value of Courant number without going implicit all the way up to the shock.
Thus, results in this case have been obtained for the value of Courant number as large as 15 . The shock stand-off distance of Fig. 6, as well as the velocity and temperature profiles of Fig. 7, compare quite well at various $\mathrm{CN}$ values. Once again, the comparison is superior over the sphertcal portion $(s=0.32)$ of the body. Figure 8 also shows good agreement between the distributions of skin-friction and heat-transfer coefficients and surface pressure employing three values of the Courant number.

The results presented here have been obtalned on the Control Data CYBER 203 vectorprocessing computer. The explicit part of the method is fully vectorized. The increase in computing time due to the addition of implicit part is about 70 percent. Typically it takes about $3.0 \times 10^{-5}$ s per mesh point per time step for the explicit part using the local minimum time step.tt With the addition of the implicit part, this time increases to about $5 \times 10^{-5} \mathrm{~s}$. Two factors which influence the computing time in the present case are the partial vectorization of the implicit part and the extent to which the implicit part is called if the shock boundary is treated explicitly. However, even with the increased computing time per time step, the total computing time is reduced significantly. In case of problem II, for example, the total computing time is about 6 times less at $\mathrm{CN}=15$ as compared to the computing time at $\mathrm{CN}=1$. With the mesh refinement parameter $\beta$ having a value of 1.02 . For a higher mesh resolution near the surface, a larger value of the Courant number may be used while still treating the shock explicitly. This would reduce the total computing time even further when compared to the computing time at $\mathrm{CN}=1$. The mesh refinement, however, is done only to the point where the mesh Reynolds number would be of order unity or so for the reasons explained earlier.

\section{Concluding Remarks}

In the analysis presented here, the recently reported implicit analog of MacCormack's earlier widely used explicit method has been extended to external axisymmetric laminar flows with strong entropy gradients. The details of the "numerics" of the implicit part are obtained in a body-oriented coordinate system with a moving outer (shock) boundary during the transient part of the solutions. The implicit analog is unconditionally stable if the boundary conditions are also specified fully implicitly (i.e. without the 1 ag of $1 / 2$ or 1 time step). this work, the Inner (wal1) boundary for the implicit part was treated through the reflection concept as suggested in the original presentation of the implicit analog by one of the present authors (RWM). The outer (shock) boundary was treated explicitly in order to avoid the specification of this boundary condition implicitly and to keep the computational algorithm simple. Thus, the present results do not contain any approximation about the treatment of the boundary condition which may affect the

FTThe same computing time per mesh point per time step for the explicit part is required if the global minimum time step is employed. However, the total computing time in this case is increased by a factor of about 2.5 . 
stability of the 1mplicit analog other than the Courant number 1imitation. The limiting values of the Courant number are provided when the shock boundary is treated explicitly. The solution algorithm outlined includes the treatment of the source term present in a weakly conservative system of equations.

For the results presented here, the Courant number in the coordinate direction along the body surface was $0(1)$, but the courant number in the direction normal to the body, because of the fine mesh-point spacing needed to resolve the viscous boundary layer at the body surface, varied from 5 to 15 depending on flow Reynolds number. A detalled comparison of various results obtained with different values of the Courant number (CN) shows that the accuracy of predictions is quite good at higher values of $\mathrm{CN}$. There is a significant saving in the overall computing time even though the computing time per time step increases by about 70 percent with the inclusion of the implicit part. The code developed for the Implicit analogue on the Control Data CYBER 203 computer was essentially an "addition" to the earlier explicit code. The coding of the implicit part 18 not affected much with the increase in complexity of the explicit part. This may have speclal appeal in the analysis of 3-D flow problems.

\section{Acknowledgment}

The authors are thankful to Dr. A. Kurmar of NASA Langley for many helpful discussions during the course of this work.

\section{Appendix A: Source Terms $\tilde{Q}_{A}$ and $\tilde{Q}_{B}$}

Based on the definftion of the total source term $Q$ given in Ref. 11, $\tilde{Q}_{A}$ and $\tilde{Q}_{B}$ identified with the coordinates $\xi$ and $n$, respectively, are defined as:

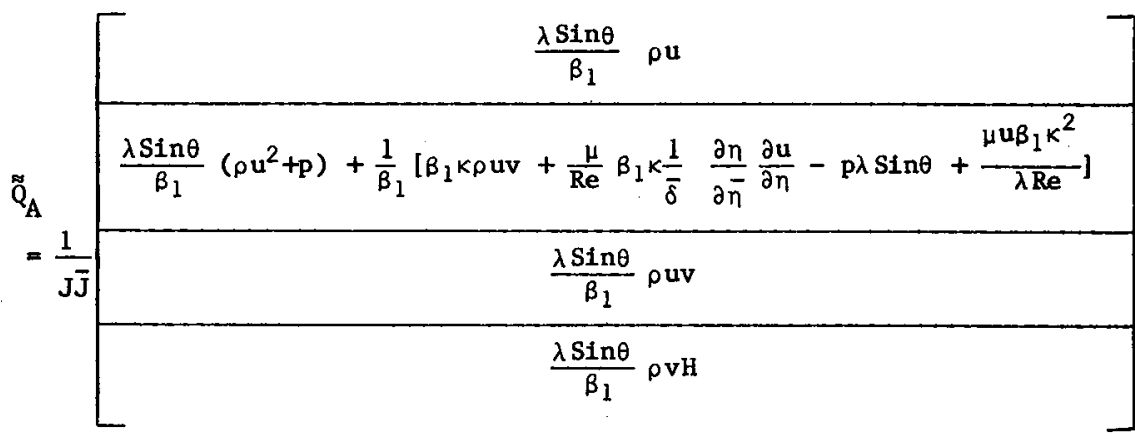

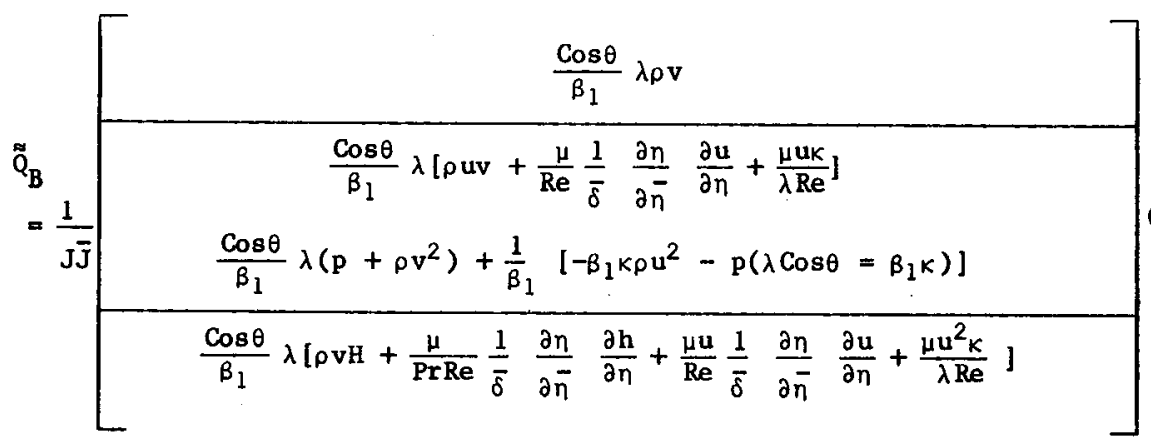

where

$$
\frac{\partial \eta}{\partial \bar{\eta}}=\frac{2 \beta}{\ell n\left(\frac{\beta+1}{\beta-1}\right)}\left(\frac{1}{\beta^{2}-\bar{\eta}^{2}}\right) ; \bar{\eta}=\beta\left[\left(\frac{\beta+1}{\beta-1}\right)^{n}-1\right] /\left[\left(\frac{\beta+1}{\beta-1}\right)^{\eta}+1\right]
$$

and the transformation Jacoblans $\mathrm{J}$ and $\overline{\mathrm{J}}$ are

$$
J=\frac{\partial(\bar{\tau}, \bar{\xi}, \bar{n})}{\partial(t, s, n)} ; \quad \bar{J}=\frac{\partial(\tau, \xi, n)}{\partial(\bar{\tau}, \bar{\xi}, \bar{n})}
$$




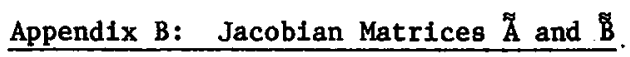

The Jacobian matrices $\AA$ and $\tilde{B}$ are obtained from

\begin{tabular}{|c|c|c|c|}
\hline$\xi_{t}$ & $\xi_{s} / \lambda$ & $\xi_{n}$ & 0 \\
\hline$-\mathrm{uu}+\frac{\alpha^{\prime} \beta^{\prime}}{\lambda} \xi_{s}+\mathrm{u}_{t}$ & $\tilde{\mathbf{u}}+\left(1-\beta^{\prime}\right) \frac{\mathbf{u}}{\lambda} \xi_{\mathrm{s}}$ & $-\frac{\beta^{\prime} v}{\lambda} \xi_{s}+u \xi_{n}$ & $\frac{\beta^{\prime}}{\lambda} \xi_{8}$ \\
\hline$-v \tilde{u}+\alpha^{\prime} \beta^{\prime} \xi_{n}+v \xi_{t}$ & $\frac{v}{\lambda} \xi_{s}-\beta^{\prime} u \xi_{n}$ & $\tilde{u}+\left(1-\beta^{\prime}\right) v \xi_{n}$ & $B^{\prime} \xi_{n}$ \\
\hline$\left(-u^{\pi}+\xi_{t}\right)\left[\left(1-\beta^{\prime}\right) \alpha^{\prime}+\frac{c^{2}}{\left.\beta^{\prime}\right]}\right.$ & $\begin{array}{l}\left(\frac{c^{2}}{\beta^{\prime}}+\alpha^{\prime}\right) \frac{\xi_{s}}{\lambda} \\
+u \beta^{\prime} \xi_{t}-u \beta^{\prime} u \tilde{u}\end{array}$ & $\begin{array}{l}\left(\frac{c^{2}}{\beta^{\prime}}+\alpha^{\prime}\right) \xi_{n} \\
+v \beta^{\prime} \xi_{t}-v \beta^{\prime} u\end{array}$ & $\begin{array}{l}\left(\beta^{\prime}+1\right) \tilde{u} \\
-\beta^{\prime} \xi_{t}\end{array}$ \\
\hline
\end{tabular}

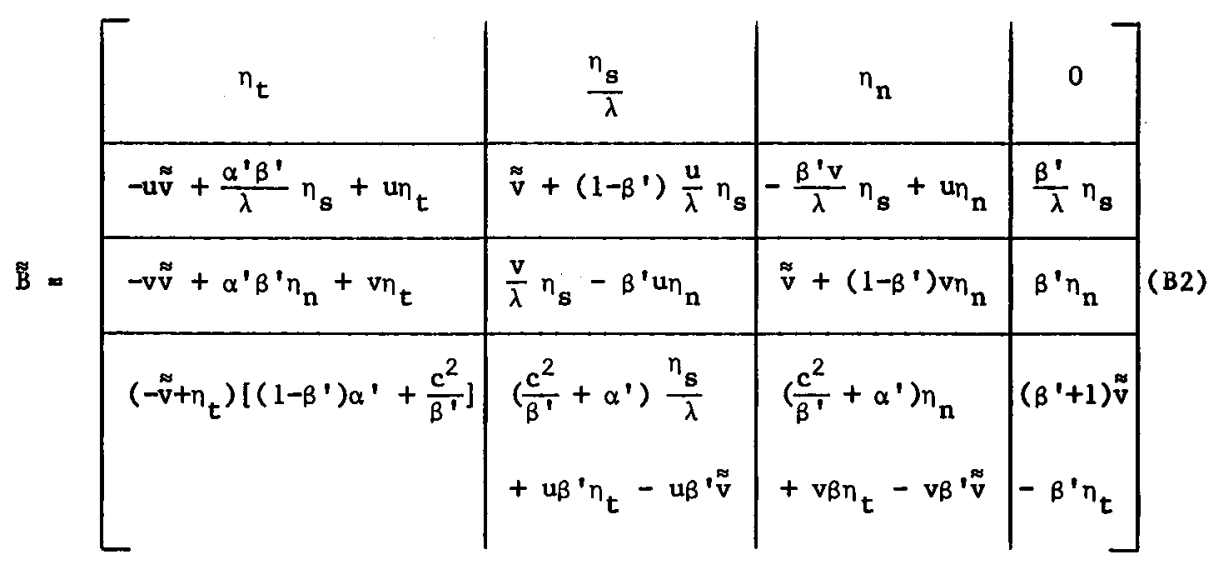

where $c=\sqrt{\gamma p / \rho}$ 1s the speed of sound, $\alpha^{\prime}=\left(u^{2}+v^{2}\right) / 2$, and $\beta^{\prime}=(\gamma-1)$.

Append1x C: Matrices $\tilde{\tilde{S}}_{\xi}, \tilde{S}_{\xi}^{-1}, \tilde{s}_{\eta}$, and $\tilde{s}_{\eta}^{-1}$

The matrices $\tilde{S}_{\xi}, \tilde{s}_{\xi}^{-1}, \tilde{S}_{\eta}$, and $\tilde{S}_{\eta}^{-1}$ are given by

$\tilde{s}_{\xi}=\left[\begin{array}{c|c|c|c}1-\frac{\alpha^{\prime} \beta^{\prime}}{c^{2}} & \frac{u \beta^{\prime}}{c^{2}} & \frac{v \beta^{\prime}}{c^{2}} & -\frac{\beta^{\prime}}{c^{2}} \\ \hline \alpha^{\prime} \beta^{\prime}-\frac{c^{2}}{d}\left(u^{\infty}-\xi_{t}\right) & \frac{c^{2}}{\lambda d} \xi_{s}-u \beta^{\prime} & \frac{c^{2}}{d} \xi_{n}-v \beta^{\prime} & \beta^{\prime} \\ \hline \frac{\left(u c \xi_{n}-v c \xi_{s} / \lambda\right)}{\rho d} & -\frac{c}{\rho d} \xi_{n} & \frac{c}{\lambda \rho d} \xi_{s} & 0 \\ \hline \alpha^{\prime} \beta^{\prime}+\frac{c^{2}}{d}\left(v-n_{t}\right) & -u \beta^{\prime}-\frac{c^{2}}{\lambda d} \xi_{s} & -v \beta^{\prime}-\frac{c^{2}}{d} \xi_{n} & \beta^{\prime}\end{array}\right]$




$\left.\begin{array}{c|c|c|c|}1 & \frac{1}{2 c^{2}} & 0 & \frac{1}{2 c^{2}} \\ \hline u & \frac{\left(u+\frac{c^{2}}{\lambda d} \xi_{s}\right)}{2 c^{2}} & -\frac{\rho c}{d} \xi_{n} & \frac{\left(u-\frac{c^{2}}{\lambda d} \xi_{s}\right)}{2 c^{2}} \\ \hline v & \frac{\left(\nu+\frac{c^{2}}{d} \xi_{n}\right)}{2 c^{2}} & \frac{\rho c}{\lambda d} \xi_{s} & \frac{\left(v-\frac{c^{2}}{d} \xi_{n}\right)}{2 c^{2}} \\ \hline \alpha^{\prime} & \frac{\left[\alpha^{\prime}+\frac{c^{2}}{d}\left(\tilde{u}_{t}-\xi_{t}\right)\right]}{2 c^{2}} & \frac{\rho c}{d}\left(-u \xi_{n}+\frac{v}{\lambda} \xi_{s}\right) & \frac{\left[\alpha^{\prime}-\frac{c^{2}}{d}\left(\tilde{u}_{-\xi_{t}}\right)\right]}{2 c^{2}} \\ & +\frac{1}{2 \beta^{\prime}} & & \frac{1}{2 \beta^{\prime}}\end{array}\right]$

$S_{n}=\left[\begin{array}{c|c|c|c}\frac{u \beta^{\prime}}{c^{2}} & \frac{v \beta^{\prime}}{c^{2}} & -\frac{\beta^{\prime}}{c^{2}} \\ \hline \frac{\left(v c n_{s} / \lambda-u c n_{n}\right)}{\rho g} & \frac{c}{\rho g} n_{n} & -\frac{c}{\lambda \rho g} n_{s} & 0 \\ \hline \alpha^{\prime} \beta^{\prime}-\frac{c^{2}}{g}\left(\tilde{v}-n_{t}\right) & \frac{c^{2}}{\lambda g} n_{s}-u \beta^{\prime} & \frac{c^{2}}{g} n_{n}-v \beta^{\prime} & \beta^{\prime} \\ \hline \alpha^{\prime} \beta^{\prime}+\frac{c^{2}}{g}\left(\tilde{v}-n_{t}\right) & -\frac{c^{2}}{\lambda g} n_{s}-u \beta^{\prime} & -\frac{c^{2}}{g} n_{n}-v \beta^{\prime} & \beta^{\prime}\end{array}\right]$

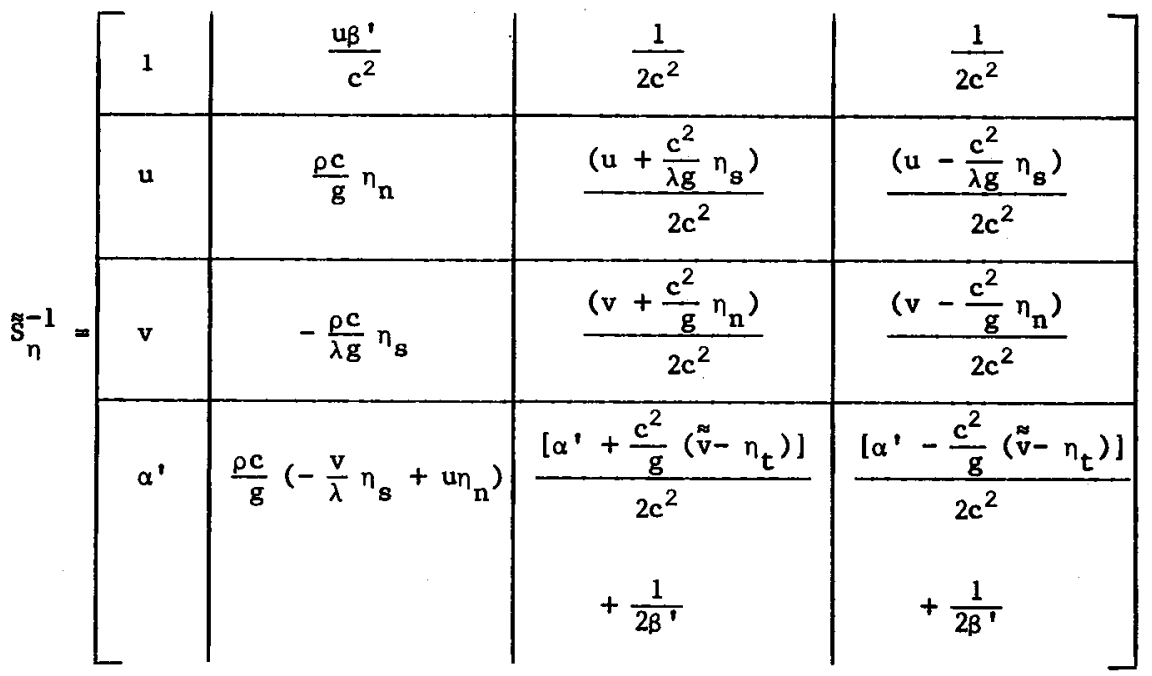

If it is assumed that the outer boundary (which is shock in the present case and moves with time during the transient part of the solutions) 18 fixed, for example for the internal flow problems, then $\eta_{t}$ and $\xi_{t}$ are Identically zero and Eqs. (C1) through (C4) reduce to those obtained in Ref. 7 for a flat surface $(\lambda=1)$. It may be mentioned that for obtaining the expressions provided here certain columns and rows of Eqs. (12) and (13) of Ref. 7 need to be multiplied and divided by ( $\rho a \sqrt{2}$ ) and a change in their order 18 required. 


\section{References}

${ }^{1}$ Beam, R. M., and Warming, R. F., "An Implicit Finite-Difference Algorithm for Hyperbolic Systems in Conservation Law Form," Journal of Computational Physics, Vol. 22, pp. 87-110, 1976.

${ }^{2}$ Briley, W. R., McDonald, H., and Gribeling, H. J., "Solution of the Mult1dimensional Compressible Navier-Stokes Equations by a Generalized Implicit Method," Report \#R75-911353-15, United Technologies Research Center, January 1976.

${ }^{3}$ Pulliam, T. H., and steger, J. L., "On Implicit Finite Difference Simulations of Three-Dimensional Flows," ALAA Paper 78-10, January 1978.

${ }^{4}$ MacCormack, R. W., "The Effect of Viscosity on Hypervelocity Impact Cratering," AIAA Paper 69-354, Apri1 30-May 2, 1979.

${ }^{5}$ MacCormack, R. W., "A Numerical Method for Solving the Equations of Compressible Viscous Flow," AIAA Journal, Vo1. 20, No. 9, September 1982, pp. 1275-1281.

${ }^{6}$ Kumar, A., "Some Observations on a New Numerical Method for Solving the Navier-Stokes Equations," NASA TP-1934, November 1981.

7VonLavante, E., and Thompkins, W. T., "An Implicit, Bi-Diagonal Numerical Method for Solving the Navier-Stokes Equations,:" AIAA Paper 82-0063, January 1982.

${ }^{8}$ White, M. E., and Anderson, J. D., Jr. "Application of MacCormack's Implicit Method to Quasi-One-Dimensional Nozzle Flows," AIAA Paper 82-0992, June 1982.

${ }^{9}$ Kordulla, W. and MacCormack, R. W., "Transonic Flow Compuotation Using an Explicit-Implicit Method," Proceedings of the Eighth International Conference on Numerical Methods, Aachen, Germany, June 28-July 2, 1982, pp. 286-295.

${ }^{10}$ MacCormack, R. W., "Numerical Solution of the Equations of Compressible Viscous Flow," Transonic, Shock, and Multidimensional Flows : Advances in Scientific Computing, Academic Press, Inc., 1982, pp. 161-179.

${ }^{11}$ Kumar, A., Graves, R. A., Jr., Weilmuenster, K. J., and Tiwari, S. N., "Coupled Laminar and Turbulent Flow Solutions For Jovian Entry," Aerothermodynamics and Planetary Entry, Progress in Astronautics and Aeronautics, Vol. 77 , edited by A. L. Crosbie, AIAA, New York, 1981, pp. 351-373.

S. N., "Kumar, A., Gxaves, R. A., Jr., and T1wart, S. N., "Laminar and Turbulent Flows Over a Spherically-Blunted Cone with Massive Surface Blowing," AIAA Journal, Vo1. 17, No. 12, December
${ }^{13}$ Xericos, J., and Anderson, W. A., "A Time-Dependent Approach to the Numerical Solution of the Flow Field About an Axisymmetric Vehicle at Angle of Attack," NASA CR-61982, 1968.

${ }^{14}$ Barnwe11, R. W., "A Time-Dependent Method for Calculating Supersonic Angle-of-Attack Flow About Axlsymmetric Blunt Bodles With Sharp Shoulders and Smooth Nonaxisymetric Blunt Bodies," NASA TN D-6283, August 1971.

${ }^{15}$ Gupta, R. N., Moss, J. N., and S1mmonds, A. L., "Comparison of Viscous-Shock-Layer Solutions by Time-Asymptotic and Steady-State Methods," NASA TM 84479, May 1982.

${ }^{16}$ MacCormack, R. W., and Baldwin, B. S., "A Numerical Method for Solving the Navier-Stokes Equations with Application to Shock-Boundary Layer Interactions," AIAA Paper 75-1, January 1975.

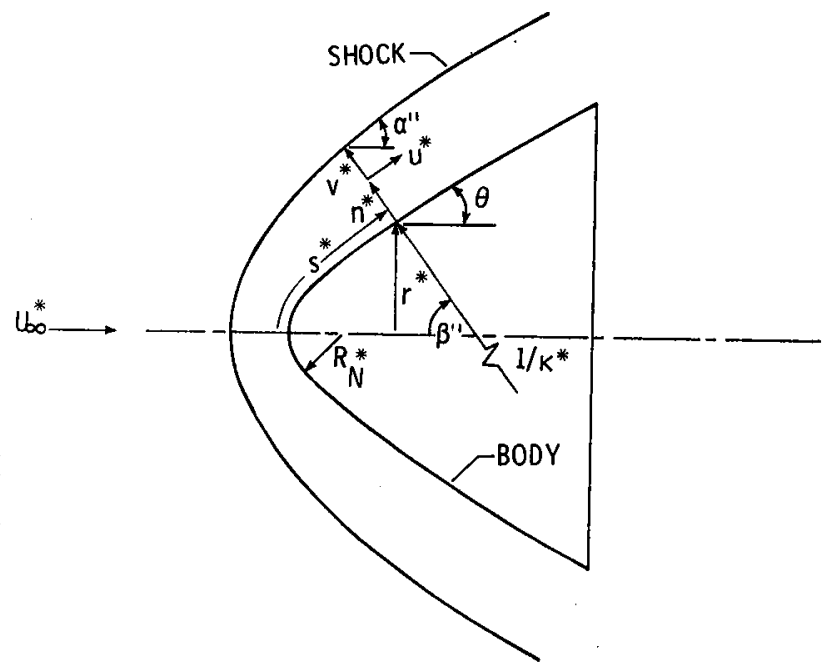

Fig. 1 Coordinate system.

(1) $\Delta t$, LOCAL MINIMUM

(2) $\Delta t_{1}=(1-0.0025 j) \Delta t_{2}$, USED HERE FOR $C N \leq 1$

(3) $\Delta t_{g}=\left[\left(\Delta t_{l}\right)_{n=0}\right]_{S p}$, GLOBAL MINIMUM FOR $C N \leq 1$

(4) $\Delta t_{3}=\operatorname{MAXIMUM}\left(\Delta t_{2} ; C_{B} \Delta t_{1}\right), \Delta t_{2}=C N(\Delta t)_{n=0} C^{\circ}=1 \mid$ USED HERE

(5) $\Delta t_{4}=\Delta t_{2}$ WITH LARGE CN

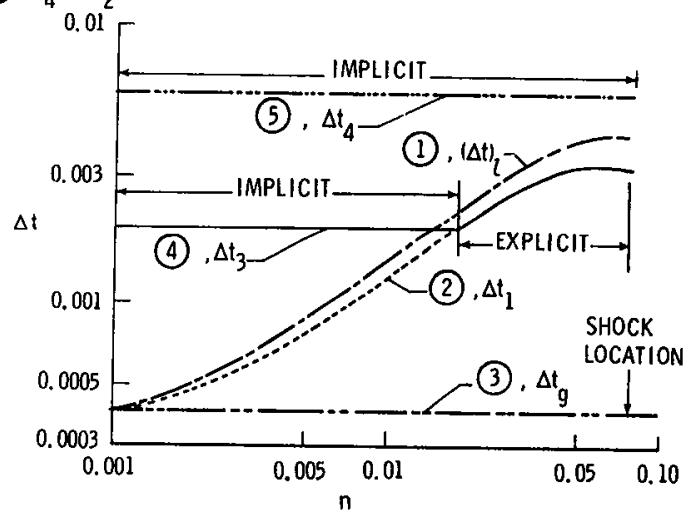

Fig. 2 Definitions of various time steps at the stagnation point for Problem II and Courant number (CN) of 5 and mesh refinement parameter $\beta=1.02$. 


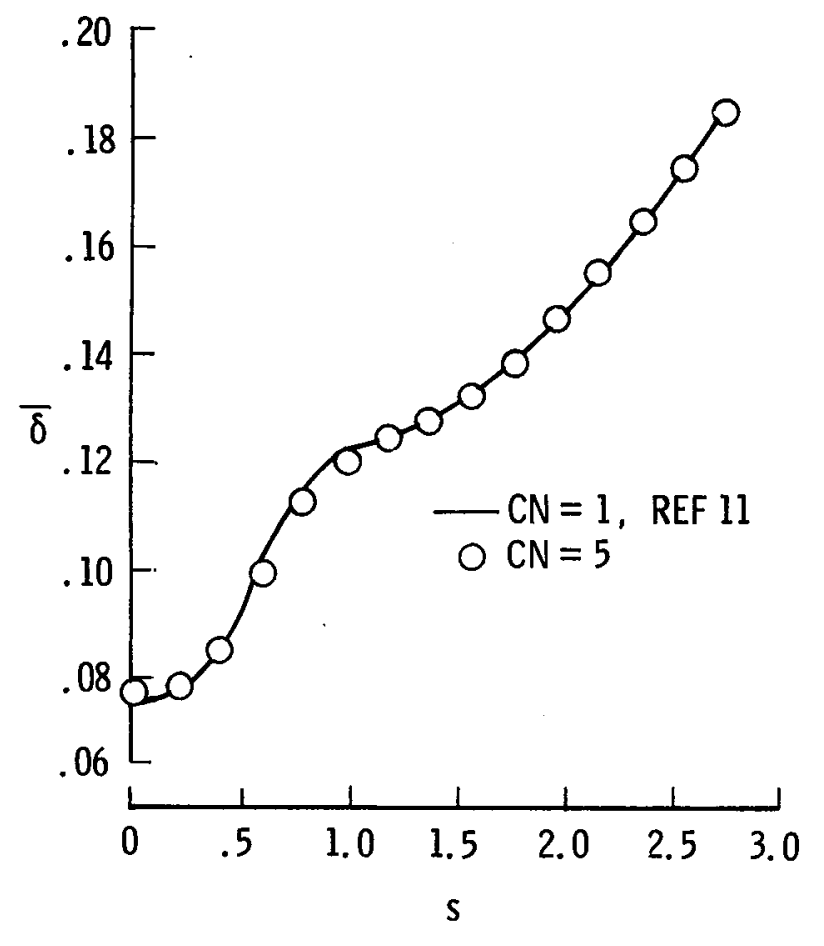

Fig. 3 Shock stand-off distance for two values of the Courant number with $\beta=1.1$, Problem I.

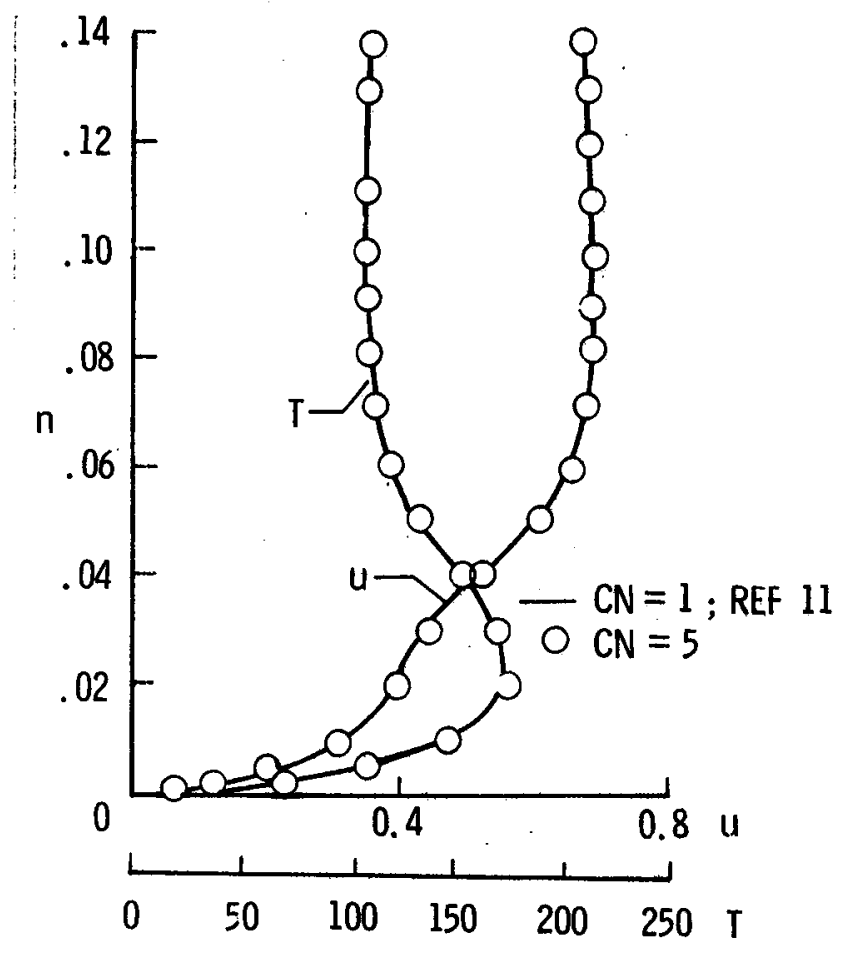

(b) $s=1.767$

F1g. 4 Concluded.

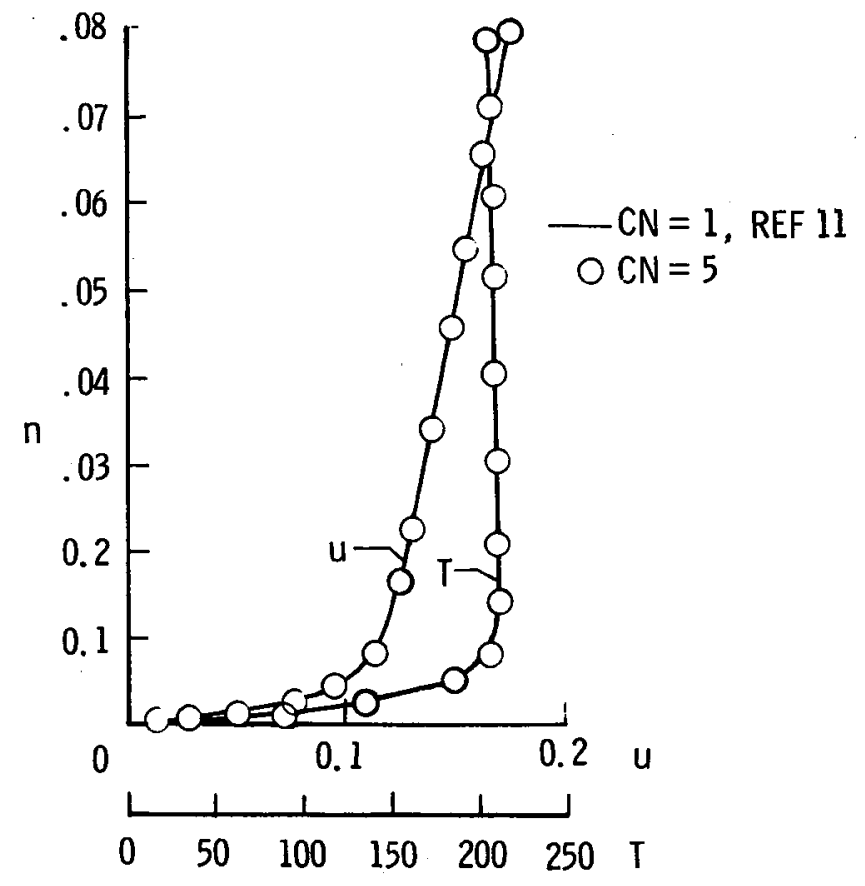

(a) $s=0.196$

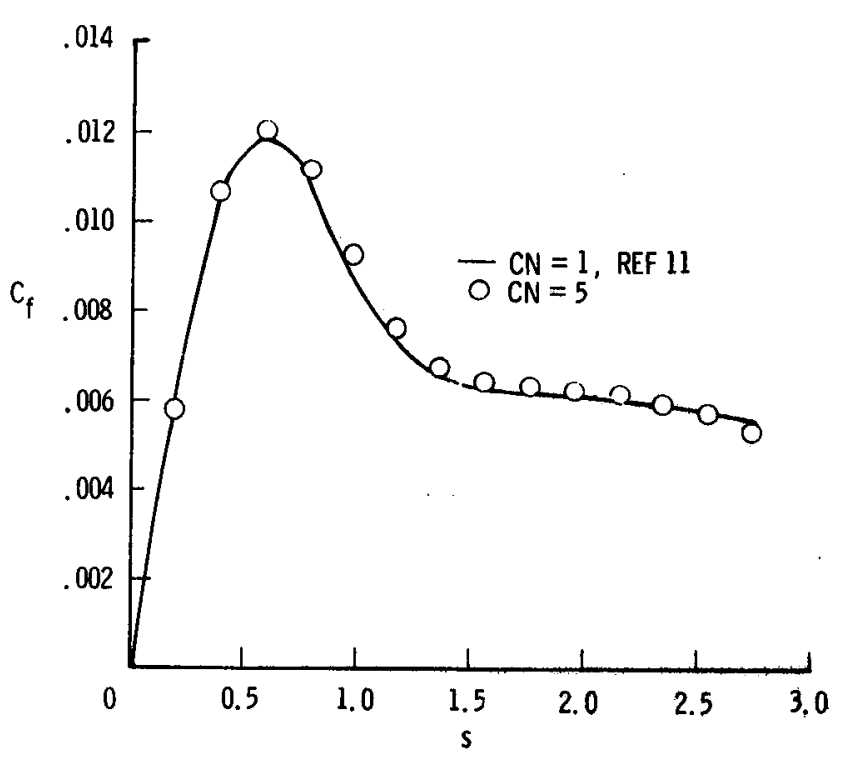

(a) SKIN-FRICTION COEFFICIENT

Fig. 5 Comparison of wall quantities with two values of the Courant number for $\beta=1.1$, Problem I.

Fig. 4 Comparison of velocity and temperature profile for two values of Courant number over the spherical portion of the body for $\beta=1.1$, Problem $I$. 


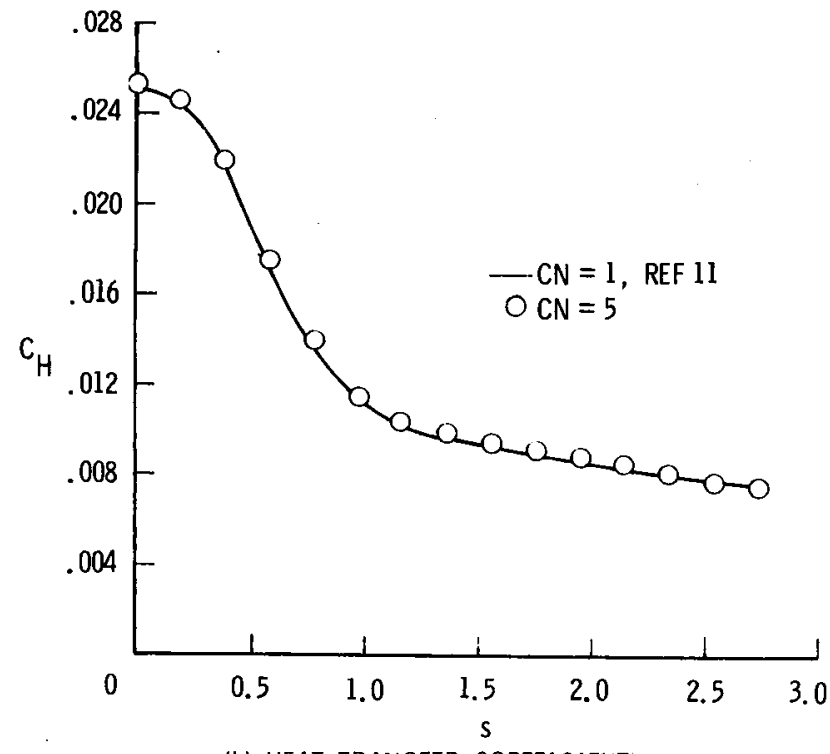

(b) HEAT TRANSFER COEFFICIENT

Fig. 5 Continued.

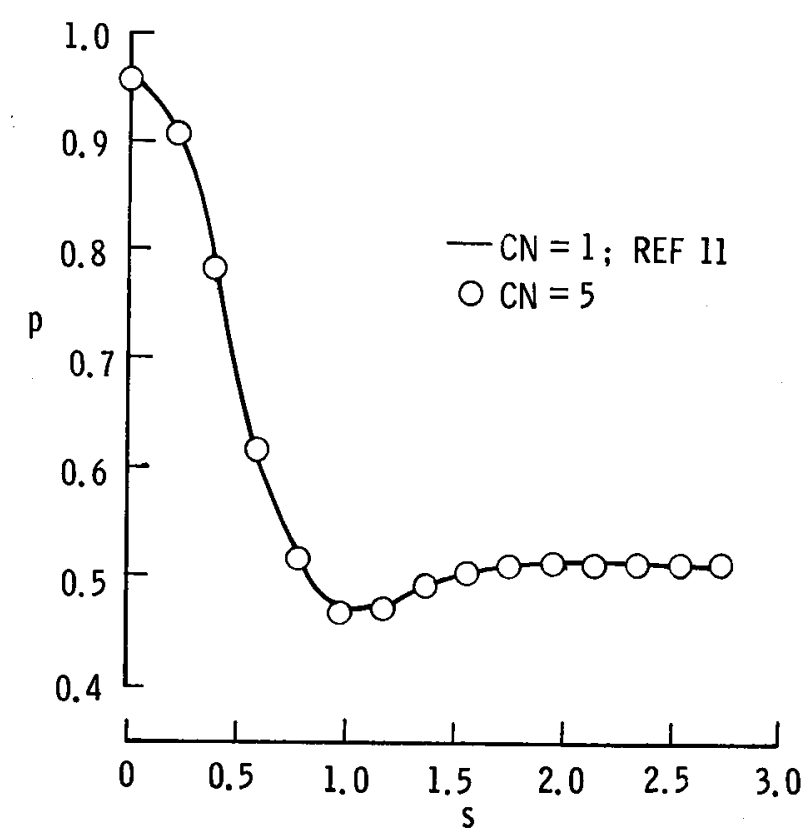

(c) SURFACE PRESSURE

Fig. 5 Concluded.

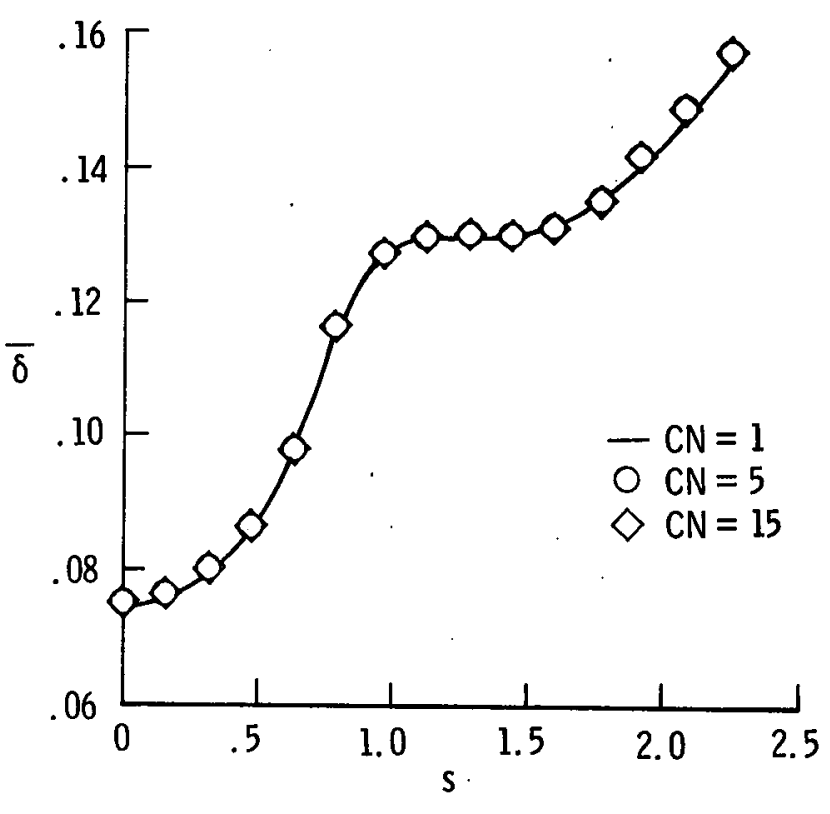

Fig. 6 Shock stand-off distance obtained with different values of the Courant number for $\beta=1.02$, Problem II.

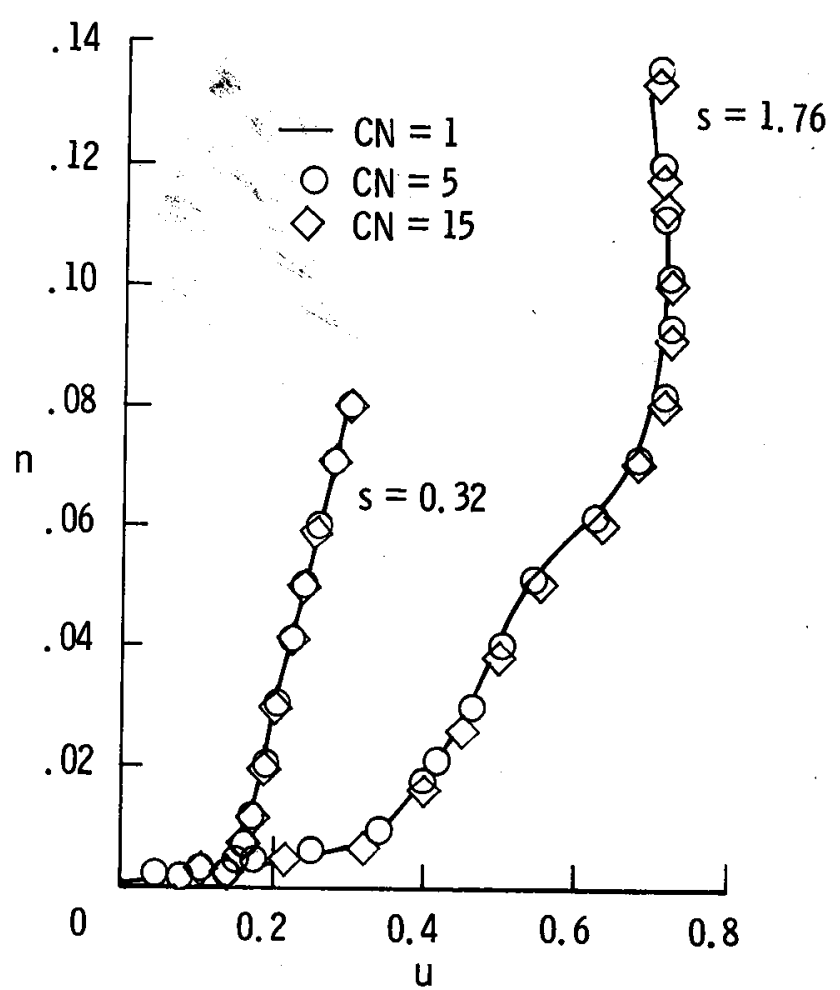

(a) TANGENTIAL VELOCITY

Fig. 7 Comparison of flowfield profiles over the spherical $(s=0.32)$ and conical flank $(s=1.76)$ portions of the body for $\beta=1.02$, Problem II. 


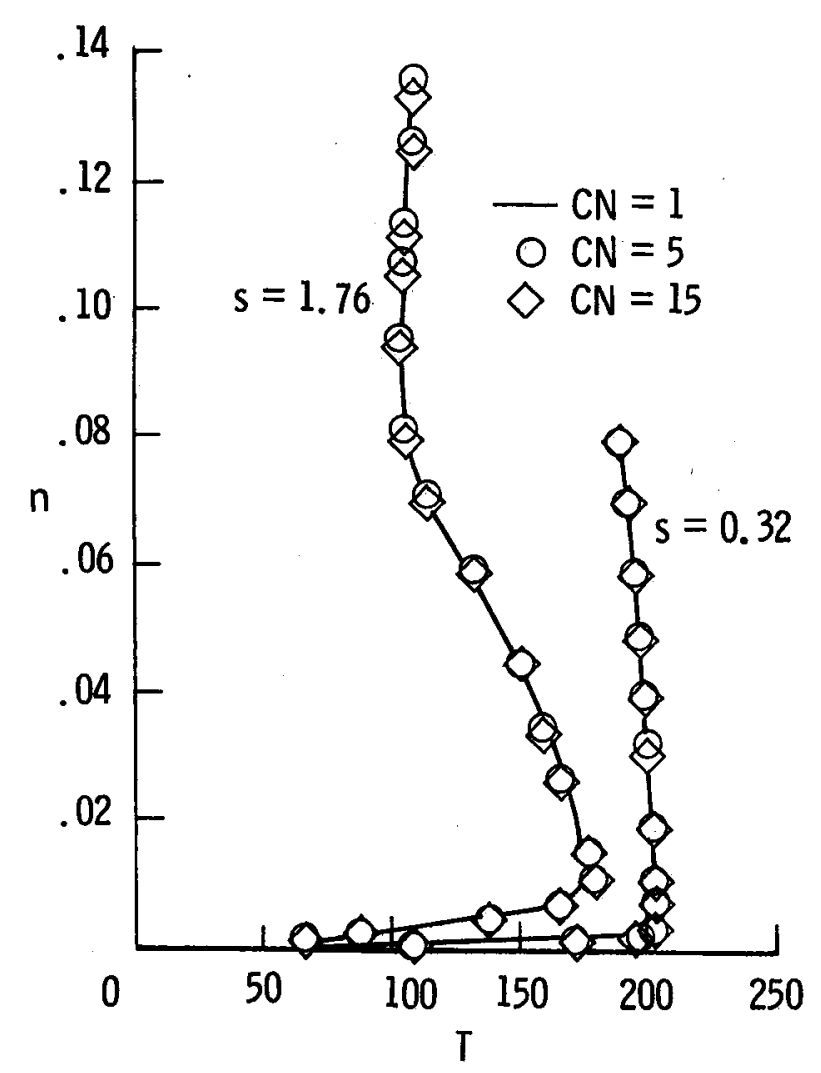

(b) TEMPERATURE

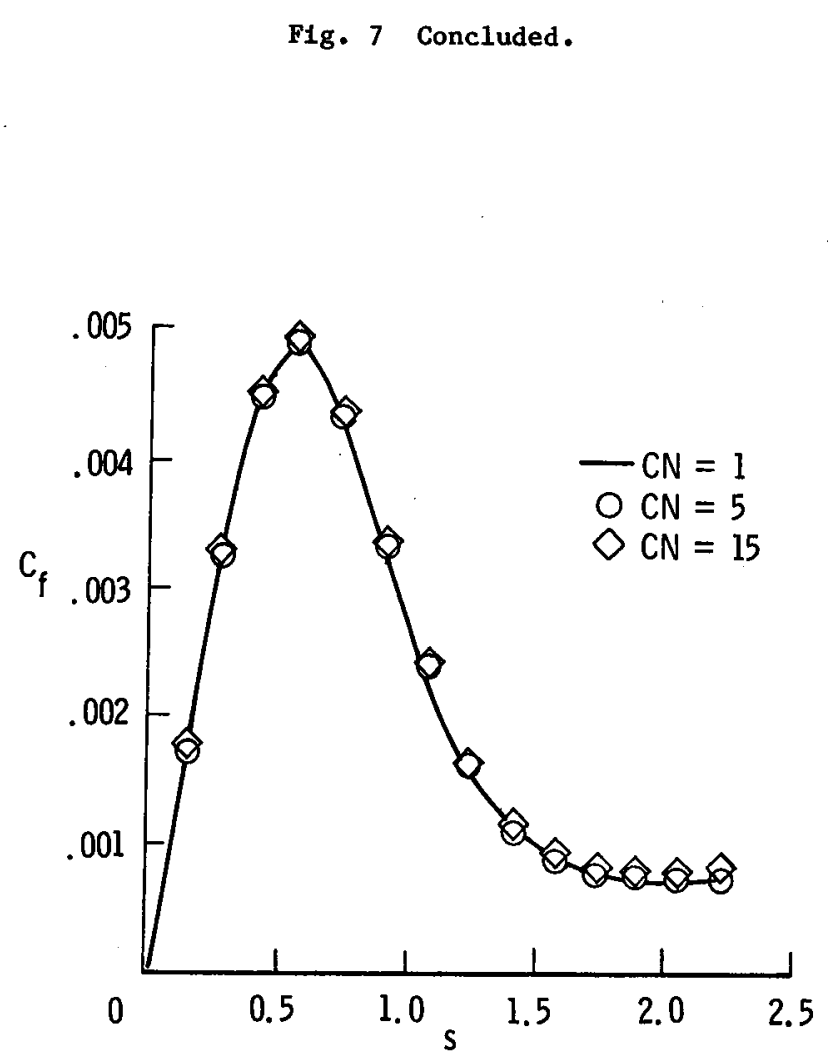

(a) SKIN-FRICTION COEFFICIENT

Fig. 8 Comparison of wall quantities with different values of the Courant number (CN) for $B=1.02$, Problem II.

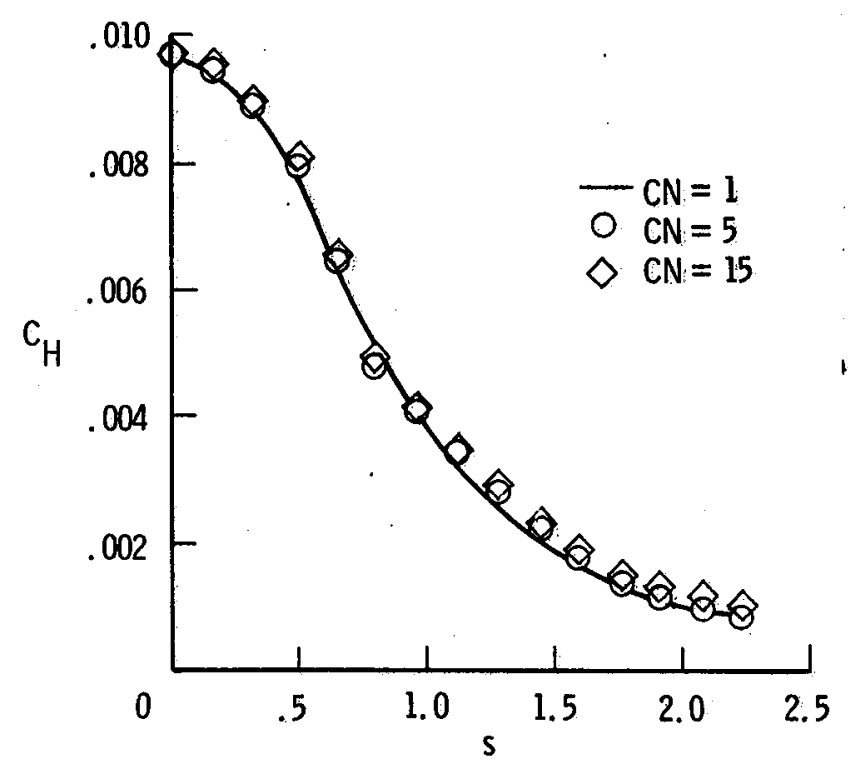

(b) HEAT TRANSFER COEFFICIENT

Fig. 8 Continued.

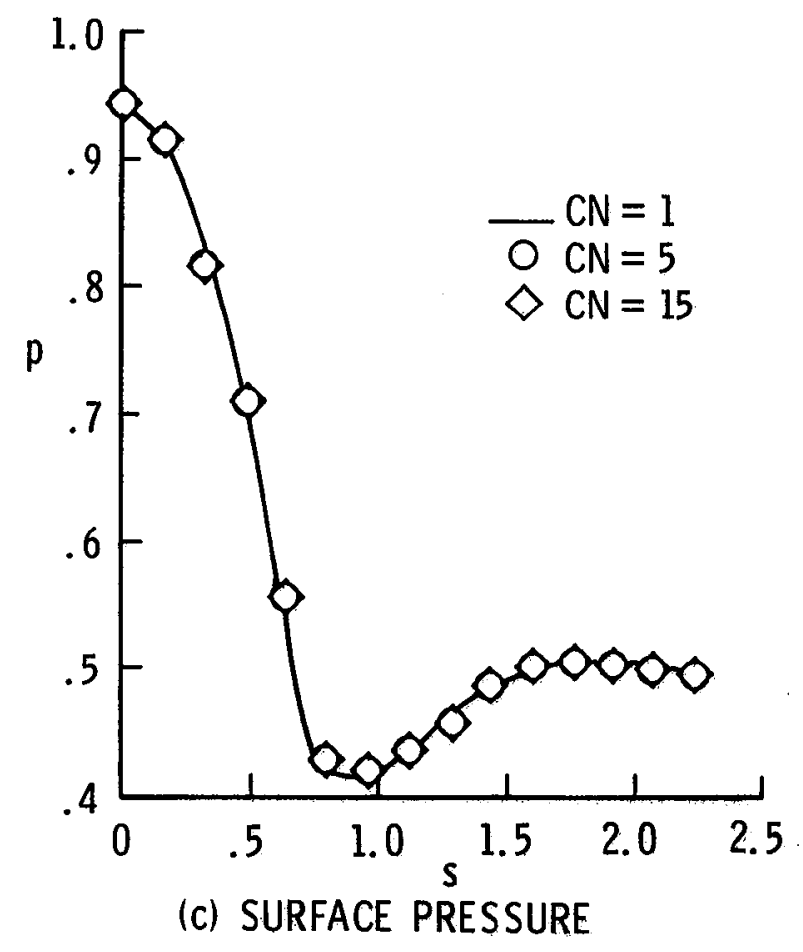

F1g. 8 Concluded. 


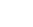




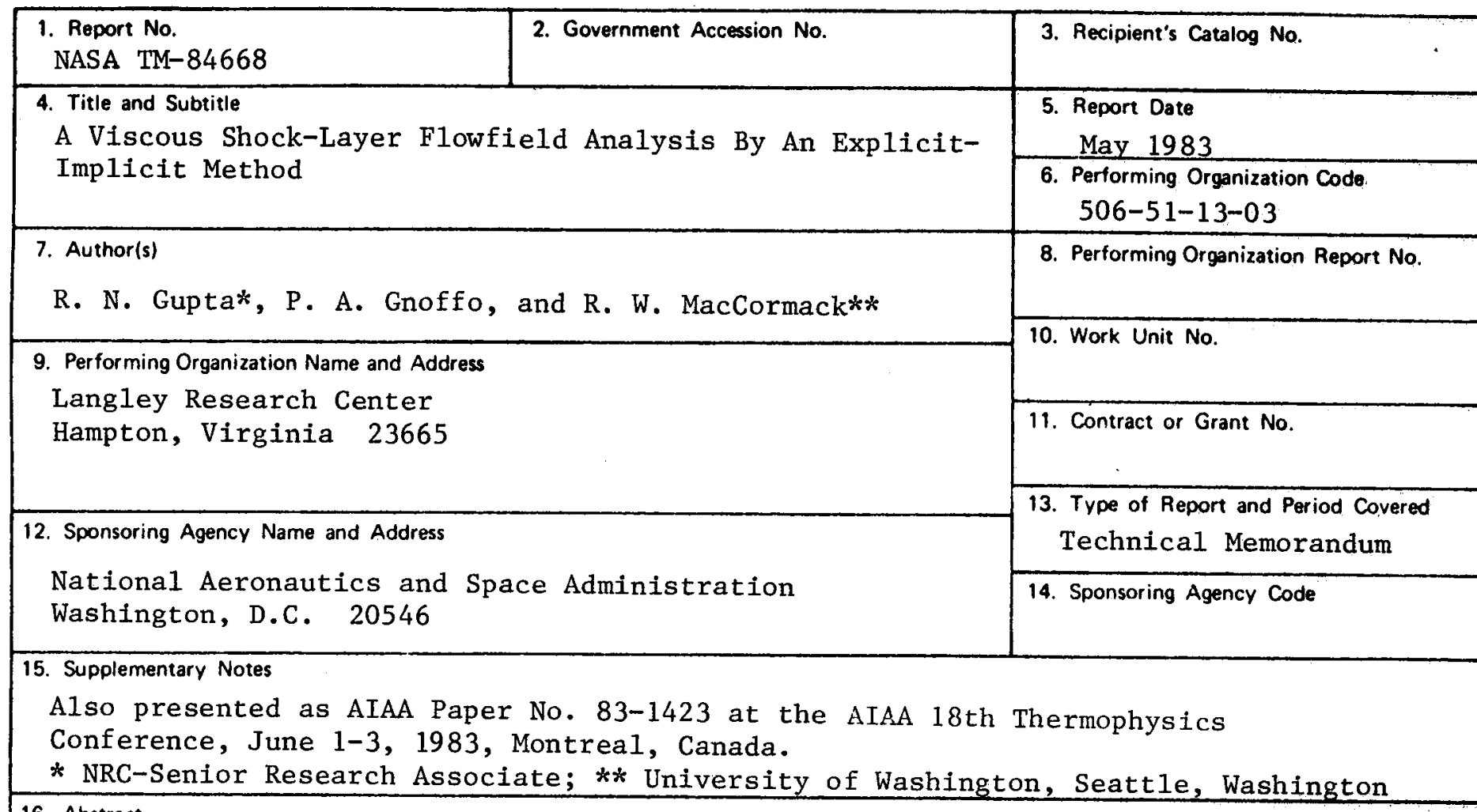

16. Abstract

The present work extends the recently reported implicit analogue of MacCormack's earlier widely used explicit method to external axisymmetric laminar flows with strong entropy gradients. The details of the "numerics" of the implicit part are provided in a body-oriented coordinate system with a moving outer (shock) boundary during the transient part of the solutions. The limiting values of the Courant number are obtained when the shock boundary is treated explicitly. The solution algorithm outlined includes the treatment of the source term associated with the equations in weak conservation form. From the results obtained for two sample problems, it becomes clear that accuracy of predictions is, indeed, very good at higher values of the Courant number. There is a significant saving in overall computing time, depending on the Courant number used and the flow Reynolds number. These properties combined with the simplicity of programing the implicit analog may appeal to researchers for using it in the analysis of 3-D flow problems.

17. Key Words (Suggested by Author(s))

Viscous-Shock-Layer

Perfect Gas

Laminar Flow

Time-Asymptotic Computations

Exp1icit-Imp1icit Computations

19. Security Classif. (of this report)

Unclassified
20. Security Classif. (of this page)

Unclassified
Subject Category 34 


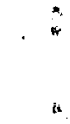

